\title{
Quantized gravitational waves in the Milne universe
}

\author{
Takahiro Tanaka and Misao Sasaki,円] \\ Department of Earth and Space Science, Graduate School of Science \\ Osaka University, Toyonaka 560, Japan
}

\begin{abstract}
The quantization of gravitational waves in the Milne universe is discussed. The relation between positive frequency functions of the gravitational waves in the Milne universe and those in the Minkowski universe is clarified. Implications to the one-bubble open inflation scenario are also discussed.
\end{abstract}

\section{INTRODUCTION}

Recently, a scenario which realizes an open universe $\left(\Omega_{0}<1\right)$ in the context of inflationary cosmology has been discussed by many authors [1 [6]. In this scenario, the flatness, homogeneity and isotropy of the universe are achieved by the accelerated expansion of the universe in the false vacuum. After a sufficiently long lapse of false vacuum inflation, the false vacuum decays into the true vacuum through quantum tunneling. This process is known as the nucleation of a vacuum bubble, which is described by the bounce solution with $O(4)$-symmetry. The bounce solution is a non-trivial solution of the field equation in Euclidean spacetime [7, 8 . The symmetry of this bubble implies the homogeneity and isotropy of the hyperbolic time-slicing inside the nucleated bubble. Thus the bubble interior becomes an open Friedmann-Robertson-Walker universe. At this stage the universe is almost empty. So in this model, the second inflation is required for entropy production.

In this context, several models of inflaton potential have been proposed [2, 4 - 6 . Now our concern is if these models are compatible with the observed anisotropies of cosmic microwave background (CMB) on large angular scales. In several recent papers 19 17, quantum fluctuations of the inflaton field which generates the initial curvature perturbations have been evaluated and the resulting spectrum of CMB anisotropies has been calculated. But in the above studies, the effects of gravity have not been fully taken into account.

There are two effects which have not been considered yet. One is the coupling between perturbations of the inflaton field and those of the metric, which may alter the spectrum of the temperature fluctuations drastically. The appearance of supercurvature modes [11,18] played a very important role in the above studies. Almost all model constraints come from the contribution of this mode. But a preliminary analysis suggests that the supercurvature mode may be sensitively affected by the effect of gravity (although the result in [12] will not be changed).

The other is the contribution of gravitational wave perturbations to the $\mathrm{CMB}$ anisotropy, which is not taken into account at all in the previous analyses. Unfortunately, our present understanding of the gravitational wave perturbation in an open inflationary universe is very poor. As has been known, a constant time hypersurface in an open inflationary universe is not a Cauchy surface of the whole spacetime [11. Thus we cannot set a commutation relation on this hypersurface when we consider quantization of a field in the open universe. This difficulty has been solved in the case of a scalar field [11, 12], but a method to handle the gravitational wave perturbation is still unclear because of the existence of gauge degrees of freedom.

In this paper, as a simple example to understand the latter effect, we consider quantization of gravitational waves on Minkowski spacetime in the context of the Milne universe. The time coordinate of the Milne universe gives the hyperbolic time-slicing of Minkowski spacetime. Despite the simplicity of this model, it turns out the model contains several important features which are essential to the understanding of gravitational wave perturbations in an open inflationary universe.

\footnotetext{
*Electronic address: tama@vega.ess.sci.osaka-u.ac.jp

† Electronic address: misao@vega.ess.sci.osaka-u.ac.jp
} 
This paper is organized as follows. In section 2 we remind the readers of the quantization scheme of a massless scalar field in the Milne universe. A method to determine the positive frequency functions in the Milne universe that describe the Minkowski vacuum state is explained. In section 3, by using analogy of the scalar case, we quantize gravitational waves in the Minkowski and Milne universes and present a candidate for the positive frequency functions in the Milne universe that describes the Minkowski vacuum state. To determine the normalization of the Milne mode functions, in Appendix, we perform canonical quantization of the gravitational wave perturbation in the Rindler universe, which is the analytic continuation of the Milne universe to the region containing a Cauchy surface. Then in section 4, we show that the Milne mode functions and the Minkowski mode functions obtained in section 3 are in fact equivalent, by explicitly constructing a unitary transformation formula between the two. In section 5 , using the results of section 3, we evaluate the temperature anisotropy caused by gravitational wave perturbations and show that it is infrared divergent in the Milne universe [19] while it is infrared finite in the Minkowski universe, though their vacuum states are equivalent. We then argue that the origin of the divergence is the unphysical setting of the problem we consider. Section 6 summarizes our results.

In this paper, we use the units, $c=\hbar=32 \pi G=1$.

\section{MASSLESS SCALAR FIELD IN THE MILNE UNIVERSE}

In order to help our understanding of the problem, we consider the quantization of a massless scalar field in the Milne universe in this section. This was discussed by diSessa 20], but we take a different approach here.

In a general background spacetime, the action of a minimally coupled real massless scalar field is given by

$$
S=-\int d^{4} x \frac{\sqrt{-g}}{2} g^{\mu \nu} \partial_{\mu} \phi \partial_{\nu} \phi
$$

where $g$ is the determinant of the metric tensor $g_{\mu \nu}$. The equation of motion for the Heisenberg operator is

$$
\square \hat{\phi}(x)=0 .
$$

We expand $\hat{\phi}(x)$ as

$$
\hat{\phi}(x)=\sum_{\Lambda}\left(\hat{a}_{\Lambda} u_{\Lambda}(x)+\hat{a}_{\Lambda}^{\dagger} \overline{u_{\Lambda}(x)}\right)
$$

by using mode functions $u_{\Lambda}(x)$ labeled by $\Lambda$ which satisfy the field equation

$$
\square u_{\Lambda}(x)=0
$$

and are normalized by the Klein-Gordon inner product as

$$
\left(u_{\Lambda}, u_{\Lambda^{\prime}}\right):=-i \int_{\Sigma} d^{3} x \sqrt{g_{\Sigma}} N^{\mu}\left(u_{\Lambda} \partial_{\mu} \overline{u_{\Lambda^{\prime}}}-\left(\partial_{\mu} \overline{u_{\Lambda}}\right) u_{\Lambda^{\prime}}\right)=\delta_{\Lambda, \Lambda^{\prime}}
$$

where $\Sigma$ is an arbitrary Cauchy surface and $N^{\mu}$ and $g_{\Sigma}$ are its unit normal and the determinant of the induced three metric on $\Sigma$, respectively. The overbar ${ }^{-}$and the dagger ${ }^{\dagger}$ represent the complex conjugate and the Hermitian conjugate, respectively. $\delta_{\Lambda, \Lambda^{\prime}}$ are to be recognized as the Kronecker delta for discrete labels and as the Dirac delta

function for continuous labels. $\hat{a}_{\Lambda}^{\dagger}$ and $\hat{a}_{\Lambda}$ are creation and annihilation operators, respectively. They satisfy the commutation relations,

$$
\left[\hat{a}_{\Lambda}, \hat{a}_{\Lambda^{\prime}}^{\dagger}\right]=\delta_{\Lambda, \Lambda^{\prime}}, \quad\left[\hat{a}_{\Lambda}, \hat{a}_{\Lambda^{\prime}}\right]=0, \quad\left[\hat{a}_{\Lambda}^{\dagger}, \hat{a}_{\Lambda^{\prime}}^{\dagger}\right]=0
$$

The vacuum state corresponding to the positive frequency function $u_{\Lambda}$ is defined by

$$
\hat{a}_{\Lambda}|0\rangle=0 \text {. }
$$

Then the two point function is expressed by the summation over modes as

$$
G^{+}\left(x, x^{\prime}\right):=\left\langle 0\left|\hat{\phi}(x) \hat{\phi}\left(x^{\prime}\right)\right| 0\right\rangle=\sum_{\Lambda} u_{\Lambda}(x) \overline{u_{\Lambda}\left(x^{\prime}\right)} .
$$




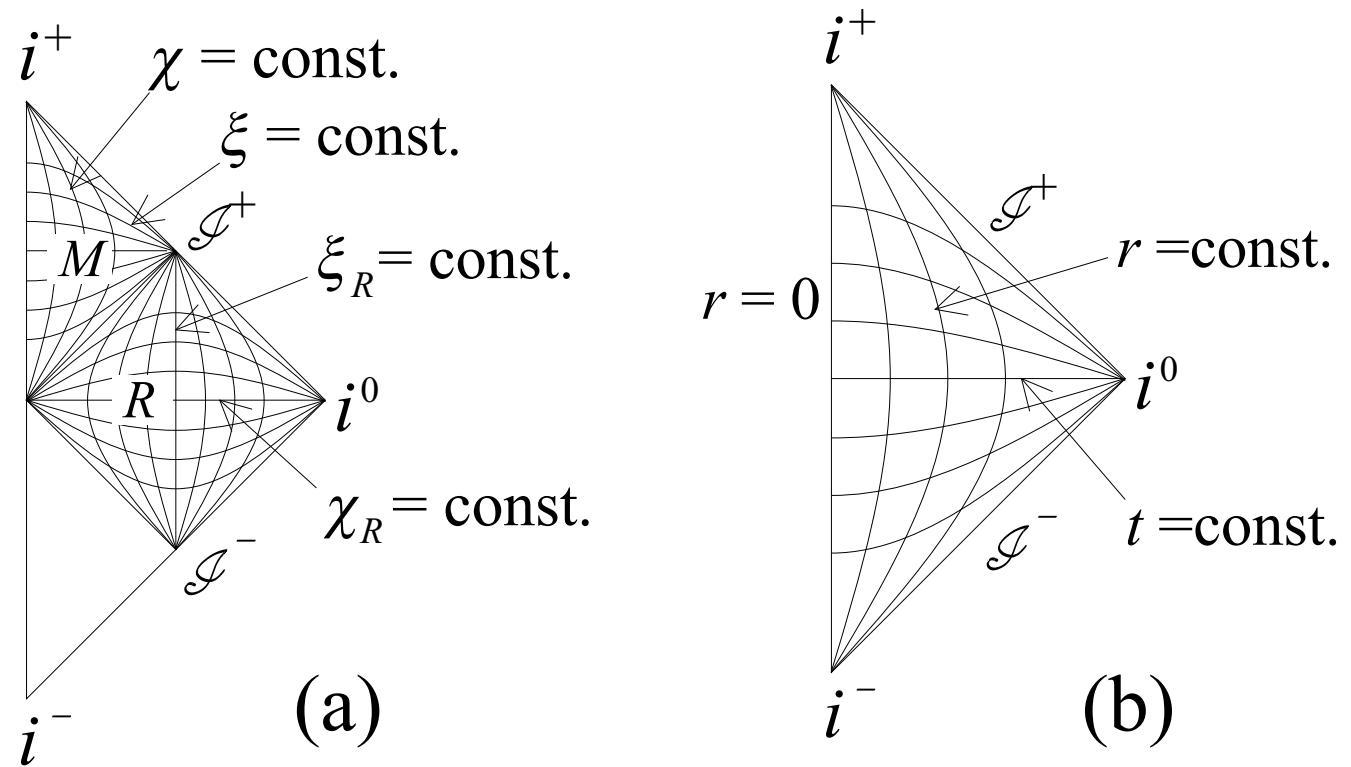

FIG. 1. Conformal diagrams of the Minkowski spacetime. The Milne coordinates and the Minkowski coordinates are shown in (a) and (b), respectively.

We introduce the coordinates which cover the whole Minkowski spacetime (which we call the Minkowski universe),

$$
d s^{2}=-d t^{2}+d r^{2}+r^{2} d \Omega^{2}
$$

and those for the Milne universe,

$$
d s^{2}=-d \xi^{2}+\xi^{2}\left(d \chi^{2}+\sinh ^{2} \chi d \Omega^{2}\right) .
$$

These two coordinates are related by

$$
t=\xi \cosh \chi, \quad r=\xi \sinh \chi .
$$

The Milne coordinates do not cover the whole region of Minkowski spacetime but only the interior of the futuredirected light cone emanating from the origin $t=r=0$. This feature is displayed in Fig. 1 by using a conformal diagram. If one tries to quantize a field in the Milne universe, one finds the $\xi=$ constant hypersurface is of no use for setting canonical commutation relations because it is not a Cauchy surface of the whole spacetime. So the extension of the Milne coordinates over the light cone to the uncovered region must be considered. The region covered by a natural extension of the Milne coordinates is known as the (spherical) Rindler universe. The Rindler coordinates are introduced by the extension of the Milne coordinates as

$$
\chi_{R}=\chi \mp \frac{\pi}{2} i, \quad \xi_{R}= \pm i \xi
$$

and the metric becomes

$$
d s^{2}=d \xi_{R}^{2}+\xi_{R}^{2}\left(-d \chi_{R}^{2}+\cosh ^{2} \chi_{R} d \Omega^{2}\right)
$$

To determine which sign of this extension one should take, we proceed as follows. When one approaches the light cone $t=r$ for a fixed $r, t=r \operatorname{coth} \chi \rightarrow r\left(1+2 e^{-2 \chi}\right)$ as $\chi \rightarrow+\infty$. Requiring the analyticity on the lower half complex $t$-plane, which is the nature of positive frequency functions of the Minkowski vacuum state, we must go round the point $t=r$ clockwise in the complex $t$-plane. Hence we choose the upper sign.

The positive frequency functions for the Minkowski vacuum are given in the Minkowski coordinates as

$$
u_{k \ell m}=U_{k}(t) \Psi_{k \ell}(r) Y_{\ell m}(\Omega)
$$

where

$$
U_{k}(t)=\sqrt{\frac{1}{2 k}} e^{-i k t}
$$




$$
\Psi_{k \ell}(r)=\sqrt{\frac{2}{\pi}} k j_{\ell}(k r),
$$

with $Y_{\ell m}(\Omega)$ being the spherical harmonics on the unit 2-sphere and $j_{\ell}(x)$ the $\ell$ th order spherical Bessel function. We adopt the phase convention of the spherical harmonics such that $\overline{Y_{\ell m}}=Y_{\ell-m}$. Here we emphasize that $k$ is positive.

To find positive frequency functions for the Minkowski vacuum written in terms of the Milne coordinates, we first solve the field equation (2.4) in the Milne coordinates. For this purpose, we introduce the harmonics on the hyperbolic 3 -space,

$$
\mathcal{P}_{p \ell}(\chi) Y_{\ell m}(\Omega)
$$

where

$$
\mathcal{P}_{p \ell}(\chi):=\frac{i^{\ell+1} \Gamma(i p+\ell+1)}{\sqrt{2}} \frac{P_{i p-\frac{1}{2}}^{-\ell-\frac{1}{2}}(\cosh \chi)}{\sqrt{\sinh \chi}},
$$

which satisfies

$$
\left[\frac{1}{\sinh ^{2} \chi} \frac{\partial}{\partial \chi}\left(\sinh ^{2} \chi \frac{\partial}{\partial \chi}\right)-\frac{\ell(\ell+1)}{\sinh ^{2} \chi}+\left(p^{2}+1\right)\right] \mathcal{P}_{p \ell}=0
$$

The phase factor $i^{\ell+1}$ is inserted in the expression for $\mathcal{P}_{p \ell}$ for later convenience. The harmonics are normalized as

$$
\int d \chi \sinh ^{2} \chi d \Omega \mathcal{P}_{p l}(\chi) Y_{l m}(\Omega) \overline{\mathcal{P}_{p^{\prime} l^{\prime}}(\chi) Y_{l^{\prime} m^{\prime}}(\Omega)}=\frac{\pi}{2 p \sinh \pi p} \delta\left(p-p^{\prime}\right) \delta_{\ell, \ell^{\prime}} \delta_{m, m^{\prime}} .
$$

Note that when $\mathcal{P}_{p \ell}$ is analytically continued to the Rindler universe by Eq. (2.12) with the upper sign, it plays the role of a positive frequency function. In the Milne universe $\mathcal{P}_{p \ell}$ is a real function except for the overall phase but, in the Rindler universe, it becomes complex in general. Then setting

$$
u_{p \ell m}(x)=\mathcal{U}_{p}(\xi) \mathcal{P}_{p \ell}(\chi) Y_{\ell m}(\Omega),
$$

the field equation (2.4) reduces to

$$
\left[\frac{\partial^{2}}{\partial \xi^{2}}+\frac{3}{\xi} \frac{\partial}{\partial \xi}+\frac{p^{2}+1}{\xi^{2}}\right] \mathcal{U}_{p}(\xi)=0
$$

The normalized solution of this equation is given by

$$
\mathcal{U}_{p}(\xi)=\frac{-i}{\sqrt{2 \pi}} e^{\pi p / 2} \xi^{-i p-1}
$$

whose analytic continuation to the Rindler universe takes the form $\mathcal{U}_{p}=\xi_{R}^{-i p-1} / \sqrt{2 \pi}$.

In fact, the Klein-Gordon inner product is evaluated on a $\chi_{R}=$ constant hypersurface in the Rindler universe as

$$
\begin{aligned}
\left(u_{p \ell m}(x), u_{p^{\prime} \ell^{\prime} m^{\prime}}(x)\right) & =i \cosh ^{2} \chi_{R}\left\{\frac{\partial \mathcal{P}_{p \ell} \overline{\mathcal{P}_{p^{\prime} \ell}}}{\partial \chi_{R}} \mathcal{P}_{p \ell} \frac{\partial \overline{\mathcal{P}_{p^{\prime} \ell}}}{\partial \chi_{R}}\right\}\left\{\frac{1}{2 \pi} \int_{0}^{\infty} \frac{d \xi_{R}}{\xi_{R}} \xi_{R}^{i\left(p-p^{\prime}\right)}\right\} \delta_{\ell, \ell^{\prime}} \delta_{m, m^{\prime}} \\
& =\delta\left(p-p^{\prime}\right) \delta_{\ell, \ell^{\prime}} \delta_{m, m^{\prime}} .
\end{aligned}
$$

Here we stress again that the analytic continuation is performed by using the relation (2.12) with the upper sign. The complex conjugate must be taken after the analytic continuation. It should also be mentioned that $\overline{u_{p \ell m}(x)} \neq$ $u_{-p \ell-m}(x)$ in the Rindler universe. This is because the lower sign in Eq. (2.12) should be used if the complex conjugate of $u_{p \ell m}$ is analytically continued to the Rindler universe. Thus there are two independent modes labeled by $\pm p$ for each value of $p^{2}$.

The equivalence of the two representations of positive frequency functions for the Minkowski vacuum (2.14) and those for the Euclidean vacuum in the Milne universe (2.20) can be directly proven by using the formula [21:22]

$$
\begin{gathered}
\int_{0}^{\infty} d k k^{i p-\frac{1}{2}} e^{-\beta k} J_{\ell+\frac{1}{2}}(\alpha k)= \\
\Gamma(i p+\ell+1) \frac{i^{\ell} e^{i \pi / 4}}{\left(\beta^{2}+\alpha^{2}\right)^{\left(i p+\frac{1}{2}\right) / 2}} P_{i p-\frac{1}{2}}^{-\ell-\frac{1}{2}}\left(\frac{\beta}{\left(\beta^{2}+\alpha^{2}\right)^{1 / 2}}\right) ; \\
-\frac{\pi}{2}<\arg \alpha<\pi, \quad \operatorname{Re} \beta>|\operatorname{Im} \alpha| .
\end{gathered}
$$


Identifying $\alpha$ with $r$ and $\beta$ with it and assuming the existence of a small imaginary part in $t$ as $t-i \epsilon$, the above formula gives a unitary transformation relation of the scalar modes,

$$
\int_{0}^{\infty} d k C_{k p} U_{k} \Psi_{k \ell}=\mathcal{U}_{p} \mathcal{P}_{p \ell}
$$

where

$$
C_{k p}=\frac{1}{\sqrt{2 \pi}} k^{i p-\frac{1}{2}}
$$

Further the inverse transformation is given by

$$
\int_{-\infty}^{\infty} d p \overline{C_{k p}} \mathcal{U}_{p} \mathcal{P}_{p \ell}=U_{k} \Psi_{k \ell}
$$

It is instructive to calculate the following quantity similar to the Klein-Gordon inner product on the $\xi=$ constant hypersurface in the Milne universe,

$$
-i \xi^{3} \int \sinh ^{2} \chi d \chi d \Omega\left\{\frac{\partial u_{p \ell m}}{\partial \xi} \overline{u_{p^{\prime} \ell^{\prime} m}}-u_{p \ell m} \frac{\partial \overline{u_{p^{\prime} \ell^{\prime} m}}}{\partial \xi}\right\}=\frac{e^{\pi p}}{2 \sinh \pi p} \delta\left(p-p^{\prime}\right) \delta_{\ell, \ell^{\prime}} \delta_{m, m^{\prime}} .
$$

Since the hypersurface is not a Cauchy surface, it gives a normalization different from the correct Klein-Gordon inner product by the factor $\frac{e^{\pi p}}{2 \sinh \pi p}$.

\section{QUANTIZATION OF GRAVITATIONAL WAVES IN MINKOWSKI AND MILNE UNIVERSES}

In this section, we quantize gravitational waves in the Minkowski and Milne universes. We choose the vacuum state to be the Euclidean vacuum, i.e., the state having the property that the positive frequency functions are analytic on the lower-half complex $t$-plane. This prescription gives the usual Minkowski vacuum for the Minkowski universe for gravitational waves as well. We expect the same is true for the Milne universe. The equivalence of thus chosen vacuum for the Milne universe with the Minkowski vacuum will be explicitly shown in the next section.

We write the metric perturbation as

$$
h_{\mu \nu}=g_{\mu \nu}-\eta_{\mu \nu}
$$

where $\eta_{\mu \nu}$ is the background metric. The action for $h_{\mu \nu}$ is given by

$$
S_{G W}=\frac{1}{2} \int d^{4} x \sqrt{-\eta}\left(-h_{\mu \nu ; \rho} h^{\mu \nu ; \rho}+2 h_{\mu \nu ; \rho} h^{\rho \mu ; \nu}-2 h_{\mu \nu} ; \nu h^{; \mu}+h_{; \mu} h^{; \mu}\right),
$$

where $h=h_{\mu}^{\mu}$. We denote the quantum counterpart of $h_{\mu \nu}$ by $\hat{h}_{\mu \nu}$.

\section{A. The case of the Minkowski universe}

First we consider quantization of gravitational waves in the Minkowski universe expressed in terms of the coordinates (2.9). As usual, we work in the traceless Lorentz gauge,

$$
h_{; \nu}^{\mu \nu}=0, \quad h_{\mu}^{\mu}=0 .
$$

where semicolon is the covariant derivative with respect to the background metric. As these conditions do not fix the gauge completely, we impose an additional condition,

$$
h_{t \mu}=0 \text {. }
$$

To quantize $h_{\mu \nu}$, we decompose it in terms of tensor harmonics on the flat Euclidean 3-space,

$$
\begin{aligned}
h_{\mu \nu} & =h_{\mu \nu}^{(e)}+h_{\mu \nu}^{(o)} ; \\
h_{\mu \nu}^{(e)} & =H_{(e) k \ell m}(t) G_{\mu \nu}^{(e) k \ell m}(r, \Omega), \\
h_{\mu \nu}^{(o)} & =H_{(o) k \ell m}(t) G_{\mu \nu}^{(o) k \ell m}(r, \Omega),
\end{aligned}
$$

where $G_{\mu \nu}^{(e) k \ell m}$ and $G_{\mu \nu}^{(o) k \ell m}$ are even and odd parity tensor harmonics, respectively. We then reduce the action to the one with respect to $H_{(e) k \ell m}$ and $H_{(o) k \ell m}$. In what follows, we consider even and odd parities separately. For notational simplicity, in the following discussion the indices $k, \ell, m$ will be abbreviated unless it causes any confusion. 
The even parity tensor harmonics are given by [24]

$$
\begin{aligned}
G_{t \mu}^{(e)} & =0, \\
G_{r r}^{(e)} & =T_{1}^{k \ell} Y_{\ell m}, \\
G_{r A}^{(e)} & =T_{2}^{k \ell} Y_{\ell m \| A}, \\
G_{A B}^{(e)} & =T_{3}^{k \ell} Y_{\ell m \| A B}+T_{4}^{k \ell} Y_{\ell m} \hat{\sigma}_{A B},
\end{aligned}
$$

where $\sigma_{A B} \equiv r^{2} \hat{\sigma}_{A B}$ is the metric induced on the $t, r=$ constant 2-sphere, $\hat{\sigma}_{A B}$ is the metric on the unit 2-sphere and the capital Latin indices such as $A$ and $B$ represent the projection onto this sphere; $f_{A}:=\sigma_{A} f_{\mu}$. The double vertical bar $\|$ denotes the covariant derivative with respect to $\sigma_{A B}$. Thus unless otherwise noted, we raise or lower the capital Latin indices not by $\hat{\sigma}_{A B}$ but by the metric $\sigma_{A B}$. The radial parts of the harmonics $T_{i}^{k \ell}(r)(i=1,2,3,4)$ are given by

$$
\begin{aligned}
T_{1}^{k \ell} & =\frac{1}{r^{2}} \Psi, \\
T_{2}^{k \ell} & =\frac{1}{\ell(\ell+1)}\left(\partial_{r} \Psi+\frac{1}{r} \Psi\right), \\
T_{3}^{k \ell} & =\frac{2}{(\ell-1) \ell(\ell+1)(\ell+2)} r^{2}\left(\frac{1}{r} \partial_{r} \Psi-\left\{k^{2}-\frac{\ell(\ell+1)+2}{2 r^{2}}\right\} \Psi\right) \\
& =\frac{2}{(\ell-1) \ell(\ell+1)(\ell+2)} r^{2}\left(\partial_{r}^{2} \Psi+\frac{3}{r} \partial_{r} \Psi-\frac{\ell(\ell+1)-2}{2 r^{2}} \Psi\right), \\
T_{4}^{k \ell} & =\frac{1}{(\ell-1)(\ell+2)} r^{2}\left(\frac{1}{r} \partial_{r} \Psi-\left\{k^{2}-\frac{2}{r^{2}}\right\} \Psi\right) \\
& =\frac{1}{(\ell-1)(\ell+2)} r^{2}\left(\partial_{r}^{2} \Psi+\frac{3}{r} \partial_{r} \Psi-\frac{\ell(\ell+1)-2}{r^{2}} \Psi\right),
\end{aligned}
$$

where $\Psi$ is defined in Eq. (2.15) and we used the equation satisfied by $\Psi$;

$$
\left[\frac{1}{r^{2}} \partial_{r} r^{2} \partial_{r}-\frac{\ell(\ell+1)}{r^{2}}+k^{2}\right] \Psi=0
$$

Inserting the decomposition (3.5) into the action (3.2), and using the orthogonality of the tensor harmonics [24]

$$
\int r^{2} d r d \Omega \eta^{\mu \mu^{\prime}} \eta^{\nu \nu^{\prime}} G_{\mu \nu}^{(e) k \ell m} G_{\mu^{\prime} \nu^{\prime}}^{(e) \ell^{\prime} m^{\prime}}=\frac{2 k^{4}}{(\ell-1) \ell(\ell+1)(\ell+2)} \delta\left(k-k^{\prime}\right) \delta_{\ell, \ell^{\prime}} \delta_{m, m^{\prime}}
$$

it reduces to

$$
S^{(e)}=\int d t \mathcal{L}^{(e)}
$$

where

$$
\mathcal{L}^{(e)}=\int_{0}^{\infty} d k \sum_{\ell, m} \frac{k^{4}}{(\ell-1) \ell(\ell+1)(\ell+2)}\left(\left|\partial_{t} H_{(e) k \ell m}(t)\right|^{2}-k^{2}\left|H_{(e) k \ell m}(t)\right|^{2}\right)
$$

Note that because of the reality of $h_{\mu \nu}$, one has

$$
\overline{H_{(e) k \ell m}}=H_{(e) k \ell-m}
$$

Then the field equation reduces to

$$
\left[\frac{\partial^{2}}{\partial t^{2}}+k^{2}\right] H_{(e) k \ell m}(t)=0
$$


and thus the solution is given by

$$
H_{(e) k \ell m}(t) \propto U_{k}(t)
$$

where $U_{k}(t)$ is defined in Eq. (2.15).

Now we consider the quantization. We write the field operator as

$$
\begin{aligned}
\hat{h}_{\mu \nu}^{(e)} & =\int_{0}^{\infty} d k \sum_{\ell, m}\left(N_{(e) k \ell m} U_{k}(t) G_{\mu \nu}^{(e) k \ell m} \hat{a}_{(e) k \ell m}+\text { h.c. }\right) \\
& =\int_{0}^{\infty} d k \sum_{\ell, m}\left(N_{(e) k \ell m} U_{k}(t) \hat{a}_{(e) k \ell m}+\overline{N_{(e) k \ell-m}} \overline{U_{k}(t)} \hat{a}_{(e) k \ell-m}^{\dagger}\right) G_{\mu \nu}^{(e) k \ell m},
\end{aligned}
$$

where we have used the fact that $G_{\mu \nu}^{(e) k \ell m}=\overline{G_{\mu \nu}^{(e) k \ell-m}}$. The constant $N_{(e) k \ell m}$ is a normalization factor to be determined by the canonical commutation relations. Then the quantum counterpart of $H_{(e) k \ell m}$ is expressed as

$$
\hat{H}_{(e) k \ell m}=N_{(e) k \ell m} U_{k}(t) \hat{a}_{(e) k \ell m}+\overline{N_{(e) k \ell-m}} \overline{U_{k}(t)} \hat{a}_{(e) k \ell-m}^{\dagger} .
$$

Note that

$$
\hat{H}_{(e) k \ell m}^{\dagger}=\hat{H}_{(e) k \ell-m}
$$

as a quantum counterpart of Eq. (3.12). From Eq. (3.11), the canonical commutation relations to be imposed on the corresponding quantum operators are

$$
\begin{aligned}
& \frac{2 k^{4}}{(\ell-1) \ell(\ell+1)(\ell+2)}\left[\hat{H}_{(e) k \ell m}, \partial_{t} \hat{H}_{(e) k^{\prime} \ell^{\prime} m^{\prime}}^{\dagger}\right]=i \delta\left(k-k^{\prime}\right) \delta_{\ell, \ell^{\prime}} \delta_{m, m^{\prime}} \\
& {\left[\hat{H}_{(e) k \ell m}, \hat{H}_{(e) k^{\prime} \ell^{\prime} m^{\prime}}^{\dagger}\right]=0, \quad\left[\partial_{t} \hat{H}_{(e) k \ell m}, \partial_{t} \hat{H}_{(e) k^{\prime} \ell^{\prime} m^{\prime}}^{\dagger}\right]=0 .}
\end{aligned}
$$

Substituting (3.16) into the commutation relations (3.19) and using (2.6) we obtain the condition

$$
\begin{aligned}
& \frac{2 k^{4}}{(\ell-1) \ell(\ell+1)(\ell+2)}\left[\left|N_{(e) k \ell m}\right|^{2} U^{k}\left(\partial_{t} \bar{U}^{k}\right)-\left|N_{(e) k \ell-m}\right|^{2}\left(\partial_{t} U^{k}\right) \bar{U}^{k}\right]=i, \\
& \left|N_{(e) k \ell m}\right|^{2}=\left|N_{(e) k \ell-m}\right|^{2} .
\end{aligned}
$$

This implies

$$
N_{(e) k \ell m}=\frac{1}{k^{2}} \sqrt{\frac{(\ell-1) \ell(\ell+1)(\ell+2)}{2}} .
$$

It should be remarked that the normalization of these modes is equivalent to setting

$$
-i \int r^{2} d r d \Omega \eta^{\mu \mu^{\prime}} \eta^{\nu \nu^{\prime}}\left(H_{\mu \nu}^{(e) k \ell m}\left(\partial_{t} \bar{H}_{\mu^{\prime} \nu^{\prime}}^{(e) k^{\prime} \ell^{\prime} m^{\prime}}\right)-\left(\partial_{t} H_{\mu \nu}^{(e) k \ell m}\right) \bar{H}_{\mu^{\prime} \nu^{\prime}}^{(e) k^{\prime} \ell^{\prime} m^{\prime}}\right)=\delta\left(k-k^{\prime}\right) \delta_{\ell, \ell^{\prime}} \delta_{m, m^{\prime}}
$$

where

$$
H_{\mu \nu}^{(e) k \ell m}=N_{(e) k \ell m} U_{k}(t) G_{\mu \nu}^{(e) k \ell m}(r, \Omega)
$$

$$
\text { 2. odd parity }
$$

The odd parity tensor harmonics are given by [24]

$$
G_{t \mu}^{(o)}=0, \quad G_{r r}^{(o)}=0
$$




$$
\begin{aligned}
G_{r A}^{(o)} & =T_{5}^{k \ell} \mathcal{Y}_{A}, \\
G_{A B}^{(o)} & =2 T_{6}^{k \ell} \mathcal{Y}_{A B},
\end{aligned}
$$

where

$$
\mathcal{Y}_{A}:=Y_{\| C} \hat{\epsilon}_{A}^{C}, \quad \mathcal{Y}_{A B}:=Y_{\| C(A} \hat{\epsilon}_{B)}^{C}=\ell(\ell+1) Y_{C(A} \hat{\epsilon}_{B)}^{C},
$$

with $\hat{\epsilon}_{A B}$ being the unit anti-symmetric tensor on the unit 2 -sphere $\left(\hat{\epsilon}_{\theta \varphi}=\sin \theta\right.$ etc. $)$ and $\hat{\epsilon}^{A}{ }_{B}=\hat{\sigma}^{A C} \hat{\epsilon}_{C B}$. The radial parts of the harmonics are given by

$$
T_{5}^{k \ell}=\Psi, \quad T_{6}^{k \ell}=\frac{1}{\ell(\ell+1)-2} \partial_{r} r^{2} \Psi .
$$

Again, inserting the decomposition (3.5) into the action (3.2), and using the orthogonality of the tensor harmonics [24.

$$
\int r^{2} d r d \Omega \eta^{\mu \mu^{\prime}} \eta^{\nu \nu^{\prime}} G_{\mu \nu}^{(o) k \ell m} G_{\mu^{\prime} \nu^{\prime}}^{(o) k^{\prime} \ell^{\prime} m^{\prime}}=\frac{2 k^{2} \ell(\ell+1)}{(\ell-1)(\ell+2)} \delta\left(k-k^{\prime}\right) \delta_{\ell, \ell^{\prime}} \delta_{m, m^{\prime}}
$$

we obtain

$$
S^{(o)}=\int d t \mathcal{L}^{(o)}
$$

where

$$
\mathcal{L}^{(o)}=\int_{0}^{\infty} d k \sum_{\ell, m} \frac{k^{2} \ell(\ell+1)}{(\ell-1)(\ell+2)}\left(\left|\partial_{t} H_{(o) k \ell m}(t)\right|^{2}-k^{2}\left|H_{(o) k \ell m}(t)\right|^{2}\right),
$$

and the reality condition implies

$$
\overline{H_{(o) k \ell m}}=H_{(o) k \ell-m} .
$$

Thus the rest of the arguments goes exactly the same as in the case of even parity if one replaces the suffix $(e)$ with $(o)$, except for the value of the normalization factor, which now is

$$
N_{(o) k \ell m}=\frac{1}{k} \sqrt{\frac{(\ell-1)(\ell+2)}{2 \ell(\ell+1)}} .
$$

\section{B. The case of the Milne universe}

We now turn to the quantization of gravitational waves in the Milne universe. Similar to the case of the Minkowski universe, one would expand $h_{\mu \nu}$ in terms of the tensor harmonics on the hyperbolic (open) 3-space to reduce the action. However, this would not give the correct normalization of the mode functions since the $\xi=$ constant hypersurface is not a Cauchy surface. Nevertheless, except for the normalization, the mode functions can be constructed by solving the classical field equation. Hence we leave aside the problem of the normalization for a moment and first solve for the mode functions expressed in terms of the tensor harmonics.

Again we choose the traceless Lorentz gauge

$$
h_{; \nu}^{\mu \nu}=0, \quad h_{\mu}^{\mu}=0 .
$$

In this gauge the field equation for the gravitational perturbation becomes

$$
h_{\mu \nu ; \alpha} ; \alpha=0 .
$$

As an additional condition to fix the gauge completely, we impose the synchronous gauge condition in the Milne coordinates (2.10),

$$
h_{\xi \mu}=0 .
$$


As is usually done in the cosmological perturbation theory, we can construct the mode functions by using the tensor harmonics, which we denote by $\mathcal{G}^{(e) p \ell m}(\chi, \Omega)$ and $\mathcal{G}^{(o) p \ell m}(\chi, \Omega)$, on the $\xi=$ constant hyperbolic 3 -space. The field operator is then expressed as

$$
\begin{aligned}
& \hat{h}_{\mu \nu}=\hat{h}_{\mu \nu}^{(e)}+\hat{h}_{\mu \nu}^{(o)} \\
& \hat{h}_{\mu \nu}^{(e)}=\int_{-\infty}^{\infty} d p \sum_{\ell, m}\left(\hat{a}_{(e) p \ell m} \mathcal{H}_{\mu \nu}^{(e) p \ell m}+\text { h.c. }\right), \\
& \hat{h}_{\mu \nu}^{(o)}=\int_{-\infty}^{\infty} d p \sum_{\ell, m}\left(\hat{a}_{(o) p \ell m} \mathcal{H}_{\mu \nu}^{(o) p \ell m}+\text { h.c. }\right),
\end{aligned}
$$

where $\mathcal{H}_{\mu \nu}^{(e) p \ell m}$ and $\mathcal{H}_{\mu \nu}^{(o) p \ell m}$ are the positive frequency functions for even and odd parity modes, respectively, for which we are going to solve below. As before, we consider the even and odd parity cases separately.

\section{1. even parity}

The even parity tensor harmonics $\mathcal{G}_{\mu \nu}^{(e) p \ell m}$ are given by [24]

$$
\begin{aligned}
\mathcal{G}_{\xi \mu}^{(e)} & =0, \\
\mathcal{G}_{\chi \chi}^{(e)} & =\mathcal{T}_{1}^{p \ell} Y_{\ell m}, \\
\mathcal{G}_{\chi A}^{(e)} & =\mathcal{T}_{2}^{p \ell} Y_{\ell m \| A}, \\
\mathcal{G}_{A B}^{(e)} & =\mathcal{T}_{3}^{p \ell} Y_{\ell m \| A B}+\mathcal{T}_{4}^{p \ell} Y_{\ell m} \hat{\sigma}_{A B} .
\end{aligned}
$$

The $\xi$-dependent radial parts are expressed in terms of the function $\mathcal{P}$ defined in Eq. (2.17) as

$$
\begin{aligned}
\mathcal{T}_{1}^{p \ell} & =\frac{1}{\sinh ^{2} \chi} \mathcal{P} \\
\mathcal{T}_{2}^{p \ell} & =\frac{1}{\ell(\ell+1)}\left(\partial_{\chi} \mathcal{P}+\operatorname{coth} \chi \mathcal{P}\right) \\
\mathcal{T}_{3}^{p \ell} & =\frac{2 \sinh ^{2} \chi}{(\ell-1) \ell(\ell+1)(\ell+2)}\left(\operatorname{coth} \chi \partial_{\chi} \mathcal{P}-\left\{p^{2}-1-\frac{\ell(\ell+1)+2}{2 \sinh ^{2} \chi}\right\} \mathcal{P}\right) \\
& =\frac{2 \sinh ^{2} \chi}{(\ell-1) \ell(\ell+1)(\ell+2)}\left(\partial_{\chi}^{2} \mathcal{P}+3 \operatorname{coth} \chi \partial_{\chi} \mathcal{P}+\left\{2-\frac{\ell(\ell+1)-2}{2 \sinh ^{2} \chi}\right\} \mathcal{P}\right) \\
\mathcal{T}_{4}^{p \ell} & =\frac{\sinh ^{2} \chi}{(\ell-1)(\ell+2)}\left(\operatorname{coth} \chi \partial_{\chi} \mathcal{P}-\left\{p^{2}-1-\frac{2}{\sinh ^{2} \chi}\right\} \mathcal{P}\right) \\
& =\frac{\sinh ^{2} \chi}{(\ell-1)(\ell+2)}\left(\partial_{\chi}^{2} \mathcal{P}+3 \operatorname{coth} \chi \partial_{\chi} \mathcal{P}+\left\{2-\frac{\ell(\ell+1)-2}{\sinh ^{2} \chi}\right\} \mathcal{P}\right)
\end{aligned}
$$

As before the indices $p, \ell$ and $m$ on $\mathcal{G}_{\mu \nu}$ and $\mathcal{P}$ are suppressed for notational simplicity.

Separating $\mathcal{H}_{\mu \nu}^{(e) p \ell m}$ by using these harmonics as

$$
\mathcal{H}_{\mu \nu}^{(e) p \ell m}=\xi^{2} \mathcal{H}_{(e) p \ell m}(\xi) \mathcal{G}_{\mu \nu}^{(e) p \ell m}(\chi, \Omega)
$$

the field equation (3.34) reduces to

$$
\left[\frac{\partial^{2}}{\partial \xi^{2}}+\frac{3}{\xi} \frac{\partial}{\partial \xi}+\frac{p^{2}+1}{\xi^{2}}\right] \mathcal{H}_{(e) p \ell m}(\xi)=0
$$

This equation is the same as Eq. (2.21). Thus the solution for $\mathcal{H}_{\mu \nu}^{(e) p \ell m}$ is given by

$$
\mathcal{H}_{\mu \nu}^{(e) p \ell m}=\mathcal{N}_{(e) p \ell m} \xi^{2} \mathcal{U}_{p} \mathcal{G}_{\mu \nu}^{(e) p \ell m},
$$


where $\mathcal{N}_{(e)}$ is a normalization constant, which is to be determined.

Now we must determine the normalization constant. To do so, in the scalar case, we analytically continued the mode functions to the Rindler universe and evaluated the Klein-Gordon norm. However, since $\mathcal{T}_{i}^{p \ell}(i=1,2,3,4)$, which would play the role of the positive frequency functions there in the present case, involve the derivatives of $\mathcal{P}$ with respect to $\chi$, one cannot single out the positive frequency functions in the Rindler universe from the present form of the mode functions. Thus one must construct a reduced action in the Rindler universe from the beginning and canonically quantize the dynamical degree of freedom there. Namely, one first expands $h_{\mu \nu}$ in terms of spherical harmonics on the 2-sphere and construct the Hamiltonian written in terms of variables which are functions of $\xi_{R}$ and $\chi_{R}$. Then imposing a gauge condition and solving constraint equations, one reduces the action to the one written in terms of a single variable, say $w_{\ell m}\left(\chi_{R}, \xi_{R}\right)$. Finally one separates this variable as $w_{p \ell m}\left(\chi_{R}\right) f_{p \ell m}\left(\xi_{R}\right)$ and rewrites the reduced action in terms of $w_{p \ell m}$ only. Canonical quantization of this variable and comparison of it with the mode functions given by Eq. (3.42) then determines the normalization factor. Since this is a complicated and tedious procedure, we defer the details to Appendix A. Here we only quote the final result,

$$
\mathcal{N}_{(e) p \ell m}=\frac{1}{p(p-i)} \sqrt{\frac{(\ell-1) \ell(\ell+1)(\ell+2)}{2}} .
$$

It is worth noting that the above normalization implies

$$
-i \cosh ^{2} \chi_{R} \int \xi_{R}^{-3} d \xi_{R} d \Omega \eta^{\mu \mu^{\prime}} \eta^{\nu \nu^{\prime}}\left(\mathcal{H}_{\mu \nu}^{(e) p \ell m}\left(\partial_{\chi_{R}} \overline{\mathcal{H}}_{\mu^{\prime} \nu^{\prime}}^{(e) \eta^{\prime} \ell^{\prime} m^{\prime}}\right)-\left(\partial_{\chi_{R}} \mathcal{H}_{\mu \nu}^{(e) p \ell m}\right) \overline{\mathcal{H}}_{\mu^{\prime} \nu^{\prime}}^{(e) p^{\prime} \ell^{\prime} m^{\prime}}\right)=\delta\left(p-p^{\prime}\right) \delta_{\ell, \ell^{\prime}} \delta_{m, m^{\prime}}
$$

which is analogous to Eq. (2.23). Although we do not know any proof, the above relation, together with the same relation in the Minkowski case (3.23), suggests that a covariant extension of this relation may hold in a general background spacetime and may be regarded as a defining relation for the norm. Furthermore the relation analogous to Eq.(2.28) also holds:

$$
-i \xi^{-1} \int \sinh ^{2} \chi d \chi d \Omega \eta^{\mu \mu^{\prime}} \eta^{\nu \nu^{\prime}}\left(\mathcal{H}_{\mu \nu}^{(e) p \ell m}\left(\partial_{\xi} \overline{\mathcal{H}}_{\mu^{\prime} \nu^{\prime}}^{(e) \ell^{\prime} \ell^{\prime} m^{\prime}}\right)-\left(\partial_{\xi} \mathcal{H}_{\mu \nu}^{(e) p \ell m}\right) \overline{\mathcal{H}}_{\mu^{\prime} \nu^{\prime}}^{(e) k^{\prime} \ell^{\prime} m^{\prime}}\right)=\frac{e^{\pi p}}{2 \sinh \pi p} \delta\left(p-p^{\prime}\right) \delta_{\ell, \ell^{\prime}} \delta_{m, m^{\prime}},
$$

The factor $\frac{e^{\pi p}}{2 \sinh \pi p}$ exactly coincides with that in the scalar case.

$$
\text { 2. odd parity }
$$

We proceed in the same way as in the case of even parity. The odd parity tensor harmonics $\mathcal{G}_{\mu \nu}^{(o)}$ are given by 24]

where

$$
\begin{aligned}
\mathcal{G}_{\xi \mu}^{(o)} & =0, \quad \mathcal{G}_{\chi \chi}^{(o)}=0, \\
\mathcal{G}_{\chi A}^{(o)} & =\mathcal{T}_{5}^{p \ell} \mathcal{Y}_{A}, \\
\mathcal{G}_{A B}^{(o)} & =2 \mathcal{T}_{6}^{p \ell} \mathcal{Y}_{A B},
\end{aligned}
$$

$$
\mathcal{T}_{5}^{p \ell}=\mathcal{P}, \quad \mathcal{T}_{6}^{p \ell}=\frac{1}{\ell(\ell+1)-2} \partial_{\chi} \sinh ^{2} \chi \mathcal{P} .
$$

As before, separating $\mathcal{H}_{\mu \nu}^{(o) p \ell m}$ as

$$
\mathcal{H}_{\mu \nu}^{(o) p \ell m}=\xi^{2} \mathcal{H}_{(o) p \ell m}(\xi) \mathcal{G}_{\mu \nu}^{(o) p \ell m}(\chi, \Omega)
$$

the field equation (3.34) reduces to

$$
\left[\frac{\partial^{2}}{\partial \xi^{2}}+\frac{3}{\xi} \frac{\partial}{\partial \xi}+\frac{p^{2}+1}{\xi^{2}}\right] \mathcal{H}_{(o) p \ell m}(\xi)=0,
$$

which is the same as in the case of even parity. The mode functions are then given by

$$
\mathcal{H}_{\mu \nu}^{(o) p \ell m}=\mathcal{N}_{(o) p \ell m} \xi^{2} \mathcal{U}_{p}(\xi) \mathcal{G}_{\mu \nu}^{(o) p \ell m}(\chi, \Omega) .
$$

After the procedure of canonical quantization as described in Appendix A, the normalization factor is found to be

$$
\mathcal{N}_{(o) p \ell m}=\frac{1}{p-i} \sqrt{\frac{(\ell-1)(\ell+2)}{2 \ell(\ell+1)}} .
$$




\section{EQUIVALENCE OF THE MILNE AND MINKOWSKI MODE FUNCTIONS}

In this section we derive a transformation formula between the gravitational wave mode functions in the Milne and Minkowski coordinates, to show the equivalence of the Milne and Minkowski mode functions. In other words, the Euclidean vacuum chosen for the Milne universe indeed turns out to be the conventional Minkowski vacuum.

Since the Minkowski mode functions $H_{\mu \nu}^{(e) k \ell m}$ and $H_{\mu \nu}^{(o) k \ell m}$ are normalized appropriately, the new mode functions defined by the unitary transformation

$$
\begin{aligned}
H_{\mu \nu}^{(e) p \ell m}: & =\int_{0}^{\infty} d k C_{k p} H_{\mu \nu}^{(e) k \ell m}, \\
H_{\mu \nu}^{(o) p \ell m} & :=\int_{0}^{\infty} d k C_{k p} H_{\mu \nu}^{(o) k \ell m},
\end{aligned}
$$

must be also suitably orthonormalized. In the following discussion, we show that these expressions coincide with the Milne mode functions $\mathcal{H}_{\mu \nu}^{(e) p \ell m}$ and $\mathcal{H}_{\mu \nu}^{(o) p \ell m}$, respectively, up to gauge. We consider even and odd parities separately.

\section{A. even parity}

First we rewrite Eq. (4.1) as

$$
\begin{aligned}
H_{\mu \nu}^{(e) p \ell m} & =\int_{0}^{\infty} d k C_{k p} N_{(e) k \ell m} U_{k}(t) G_{\mu \nu}^{(e) k \ell m}(r, \Omega) \\
& =-p(p-i) \mathcal{N}_{(e) p \ell m} \int_{-i \infty}^{t} d t^{\prime} \int_{-i \infty}^{t^{\prime}} d t^{\prime \prime} \int_{0}^{\infty} d k C_{k p} U_{k}\left(t^{\prime \prime}\right) G_{\mu \nu}^{(e) k \ell m}(r, \Omega),
\end{aligned}
$$

where we used the relation $N_{(e) k \ell m}=\mathcal{N}_{(e) p \ell m} p(p-i) / k^{2}$ and replaced the $1 / k^{2}$ factor by the double $t$-integration. Then the components of $H_{\mu \nu}^{(e) p \ell m}$ in the Milne coordinates are given in terms of those in the Minkowski coordinates as

$$
\left(\begin{array}{ccc}
H_{\xi \xi}^{(e) p \ell m} & H_{\xi \chi}^{(e) p \ell m} & H_{\xi A}^{(e) p \ell m} \\
* & H_{\chi \chi}^{(e) p \ell m} & H_{\chi A}^{(e) p \ell m} \\
* & * & H_{A B}^{(e) p \ell m}
\end{array}\right)=\left(\begin{array}{ccc}
\sinh ^{2} \chi H_{r r}^{(e) p \ell m} & \xi \sinh \chi \cosh \chi H_{r r}^{(e) p \ell m} & \sinh \chi H_{r A}^{(e) p \ell m} \\
* & \xi^{2} \cosh ^{2} \chi H_{r r}^{(e) p \ell m} & \xi \cosh \chi H_{r A}^{(e) p \ell m} \\
* & * & *
\end{array}\right)
$$

In the Minkowski coordinates, the $k$-integration in Eq. (4.3) can be performed with the aid of Eqs. (2.25), (3.6) and (3.7). Then

$$
\begin{aligned}
H_{r r}^{(e) p \ell m}= & -p(p-i) \mathcal{N}_{(e)} \frac{1}{r^{2}} \int d t \int d t \mathcal{U P} Y_{\ell m} \\
= & : \mathcal{A} Y, \\
H_{r A}^{(e) p \ell m}= & \frac{1}{\ell(\ell+1)}\left(\partial_{r}+\frac{1}{r}\right) r^{2} \mathcal{A} Y_{\| A}, \\
H_{A B}^{(e) p \ell m}= & \frac{2 r^{2}}{(\ell-1) \ell(\ell+1)(\ell+2)}\left(\partial_{r}^{2}+\frac{3}{r} \partial_{r}-\frac{\ell(\ell+1)-2}{2 r^{2}}\right) r^{2} \mathcal{A} Y_{\| A B} \\
& +\frac{\sigma_{A B}}{(\ell-1)(\ell+2)}\left(\partial_{r}^{2}+\frac{3}{r} \partial_{r}-\frac{\ell(\ell+1)-2}{r^{2}}\right) r^{2} \mathcal{A} Y .
\end{aligned}
$$

The $t$-integration in the expression for $\mathcal{A}$ can be replaced by a $\chi$-integration as follows. First we note

$$
\xi=\frac{r}{\sinh \chi}
$$

which gives

$$
d \xi=-\frac{r \cosh \chi d \chi}{\sinh ^{2} \chi},
$$

for a fixed $r$. Therefore we obtain 


$$
d t=d \xi \cosh \chi+\xi \sinh \chi d \chi=-r \frac{d \chi}{\sinh ^{2} \chi}
$$

Hence

$$
\begin{aligned}
\mathcal{A}= & -p(p-i) \mathcal{N}_{(e)} \mathcal{U}_{p}(\xi)\left[(\sinh \chi)^{-i p-1} \int d \chi(\sinh \chi)^{-2} \int d \chi(\sinh \chi)^{i p-1} \mathcal{P}_{p \ell}(\chi)\right] \\
= & -p(p-i) \mathcal{N}_{(e)} \mathcal{U}_{p}(\xi)\left[\frac{1}{(i p+\ell)(i p+\ell-1)} \frac{i^{\ell+1}}{\sqrt{2}} \frac{\Gamma(i p+\ell+1)}{(\sinh \chi)^{5 / 2}} P_{i p-\frac{5}{2}}^{-\ell-\frac{1}{2}}(\cosh \chi)\right] \\
= & \frac{-p(p-i) \mathcal{N}_{(e)} \mathcal{U}_{p}(\xi)}{(i p+\ell)(i p+\ell-1)(i p-\ell-1)(i p-\ell-2)} \\
& \times\left(2(1-i p) \operatorname{coth} \chi \frac{d}{d \chi}+2(1-i p)^{2}+\{\ell(\ell+1)-(p+i)(p+2 i)\} \frac{1}{\sinh ^{2} \chi}\right) \mathcal{P}_{p \ell}(\chi),
\end{aligned}
$$

where the formulas

$$
\partial_{\chi} \frac{(\sinh \chi)^{\nu}}{\nu-\mu} P_{\nu-1}^{\mu}(\cosh \chi)=(\sinh \chi)^{\nu-1} P_{\nu}^{\mu}(\cosh \chi)
$$

and

$$
\frac{P_{\nu}^{\mu}(\cosh \chi)}{\sqrt{\sinh \chi}}=\frac{1}{\mu+\nu+1}\left[-\sinh \chi \partial_{\chi}+\left(\nu+\frac{1}{2}\right) \cosh \chi\right] \frac{P_{\nu+1}^{\mu}(\cosh \chi)}{\sqrt{\sinh \chi}}
$$

were used in the second and third equalities, respectively. Here we mention that a derivative with respect to $r$ in the expressions (4.5) can be replaced as

$$
r \partial_{r}=-\sinh ^{2} \chi \xi \partial_{\xi}+\sinh \chi \cosh \chi \partial_{\chi}
$$

and so

$$
r \partial_{r} \mathcal{A}=\left[(1+i p) \sinh ^{2} \chi+\sinh \chi \cosh \chi \partial_{\chi}\right] \mathcal{A}
$$

The newly defined mode functions $H_{\mu \nu}^{(e) p \ell m}$ also satisfy the traceless Lorentz gauge condition (3.3), but as mentioned before it does not fix the gauge completely. In fact, $H_{\mu \nu}^{(e) p \ell m}$ satisfy the Minkowski synchronous gauge condition (3.4) but not the Milne synchronous gauge condition (3.35). Thus to compare $\mathcal{H}_{\mu \nu}^{(e) p \ell m}$ with $H_{\mu \nu}^{(e) p \ell m}$, a gauge transformation is necessary. We consider the gauge transformation

$$
\begin{aligned}
\tilde{H}_{\mu \nu}^{(e) p \ell m} & =H_{\mu \nu}^{(e) p \ell m}+\rho_{\mu ; \nu}+\rho_{\nu ; \mu} \\
& =H_{\mu \nu}^{(e) p \ell m}+g_{\mu \sigma} \partial_{\nu} \rho^{\sigma}+g_{\nu \sigma} \partial_{\mu} \rho^{\sigma}+\rho^{\sigma} \partial_{\sigma} g_{\mu \nu}
\end{aligned}
$$

so as to make $\tilde{H}_{\xi \mu}^{(e) p \ell m}=0$.

The condition $\tilde{H}_{\xi \xi}^{(e) p \ell m}=0$ gives the equation,

$$
\partial_{\xi} \rho^{\xi}=\frac{\sinh ^{2} \chi}{2} \mathcal{A} Y
$$

It is integrated to give

$$
\rho^{\xi}=\frac{\xi}{-2 i p} \sinh ^{2} \chi \mathcal{A} Y
$$

where we neglected possible existence of an integration constant which is $\xi$-independent.

The condition $\tilde{H}_{\xi \chi}^{(e) p \ell m}=0$ gives the equation

$$
\begin{aligned}
\partial_{\xi} \rho^{\chi} & =\frac{1}{\xi^{2}}\left(\partial_{\chi} \rho^{\xi}-H_{\xi \chi}^{(e) p \ell m}\right) \\
& =\frac{1}{\xi}\left(\frac{i}{2 p} \sinh ^{2} \chi \partial_{\chi}+\frac{i-p}{p} \sinh \chi \cosh \chi\right) \mathcal{A} Y
\end{aligned}
$$


and it is integrated to give

$$
\rho^{\chi}=\frac{i}{p(p-i)}\left(\frac{i}{2} \sinh ^{2} \chi \partial_{\chi}-(p-i) \sinh \chi \cosh \chi\right) \mathcal{A} Y
$$

where again we set an integration constant to zero.

The condition $\tilde{H}_{\xi A}^{(e) p \ell m}=0$ gives the equation

$$
\begin{aligned}
\partial_{\xi}\left(\hat{\sigma}_{A B} \rho^{B}\right) & =\frac{1}{r^{2}}\left(\rho_{\| A}^{\xi}-H_{\xi A}^{(e) p \ell m}\right) \\
& =\frac{1}{\xi}\left(\frac{i}{2 p}-\frac{1}{\ell(\ell+1)}\left((1+i p) \sinh ^{2} \chi+3+\sinh \chi \cosh \chi \partial_{\chi}\right)\right) \mathcal{A} Y_{\| A},
\end{aligned}
$$

and it is integrated to give

$$
\sigma_{A B} \rho^{B}=\frac{i r^{2}}{p(p-i)}\left(\frac{i}{2}-\frac{p}{\ell(\ell+1)}\left((1+i p) \sinh ^{2} \chi+3+\sinh \chi \cosh \chi \partial_{\chi}\right)\right) \mathcal{A} Y_{\| A},
$$

where once again an integration constant was set to zero.

The other components are calculated by substituting the above equations to the following formulas,

$$
\begin{aligned}
& \tilde{H}_{\chi \chi}^{(e) p \ell m}=H_{\chi \chi}^{(e) p \ell m}-2 \partial_{\chi} \rho^{\chi}+2 \xi \rho^{\xi}, \\
& \tilde{H}_{\chi A}^{(e) p \ell m}=H_{\chi A}^{(e) p \ell m}+\xi^{2} \rho_{\| A}^{\chi}+\sigma_{A B} \partial_{\chi} \rho^{B}, \\
& \tilde{H}_{A B}^{(e) p \ell m}=H_{A B}^{(e) p \ell m}+\sigma_{A C} \rho^{C}{ }_{\| B}+\sigma_{B C} \rho^{C}{ }_{\| A}+\sigma_{A B}\left(\frac{2}{\xi} \rho^{\xi}+2 \operatorname{coth} \chi \rho^{\chi}\right) .
\end{aligned}
$$

Then it is straightforward to check the equalities

$$
\tilde{H}_{\chi \chi}^{(e) p \ell m}=\mathcal{H}_{\chi \chi}^{(e) p \ell m}, \quad \tilde{H}_{\chi A}^{(e) p \ell m}=\mathcal{H}_{\chi A}^{(e) p \ell m}, \quad \tilde{H}_{A B}^{(e) p \ell m}=\mathcal{H}_{A B}^{(e) p \ell m}
$$

Thus we have shown equivalence of the positive frequency functions $H_{\mu \nu}^{(e) p \ell m}$ and $\mathcal{H}_{\mu \nu}^{(e) p \ell m}$ up to gauge.

\section{B. odd parity}

As in the case of even parity, we rewrite Eq. (4.2) as

$$
H_{\mu \nu}^{(o) p \ell m}=\int_{0}^{\infty} d k C_{k p} N_{(o) k \ell m} U_{k}(t) G_{\mu \nu}^{(o) k \ell m}(r, \Omega)=-i(p-i) \mathcal{N}_{(o) p \ell m} \int_{-i \infty}^{t} d t^{\prime} \int_{0}^{\infty} d k C_{k p} U_{k}\left(t^{\prime}\right) G_{\mu \nu}^{(o) k \ell m}(r, \Omega)
$$

Then the components of $H_{\mu \nu}^{(o) p \ell m}$ in the Minkowski coordinates are given by

$$
\begin{aligned}
H_{r A}^{(o) p \ell m} & =-i(p-i) \mathcal{N}_{(o)} \int d t \mathcal{U} \mathcal{P} \mathcal{Y}_{A} \\
& =: \mathcal{B} \mathcal{Y}_{A}, \\
H_{A B}^{(o) p \ell m} & =\frac{2}{(\ell-1)(\ell+2)} \partial_{r} r^{2} \mathcal{B} \mathcal{Y}_{A B} .
\end{aligned}
$$

Transforming the $t$-integration to a $\chi$-integration as before, $\mathcal{B}$ is evaluated as

$$
\begin{aligned}
\mathcal{B} & =i(p-i) \mathcal{N}_{(o)} \xi \mathcal{U}_{p}(\xi)\left[(\sinh \chi)^{-i p} \int d \chi(\sinh \chi)^{i p-1} \mathcal{P}_{p \ell}(\chi)\right] \\
& =i(p-i) \mathcal{N}_{(o)} \xi \mathcal{U}_{p}(\xi)\left[\frac{1}{i p+\ell} \frac{i^{\ell+1}}{\sqrt{2}} \frac{\Gamma(i p+\ell+1)}{\sqrt{\sinh \chi}} P_{i p-\frac{3}{2}}^{-\ell-\frac{1}{2}}(\cosh \chi)\right] \\
& =\frac{-i(p-i) \mathcal{N}_{(o)} \xi \mathcal{U}_{p}(\xi)}{(i p+\ell)(i p-\ell-1)}\left[\sinh \chi \partial_{\chi}-(i p-1) \cosh \chi\right] \mathcal{P}_{p \ell}(\chi) .
\end{aligned}
$$

As in the even parity case, by using Eq. (4.12), we can rewrite the derivative with respect to $r$ in (4.24) as 


$$
\partial_{r} r^{2} \mathcal{B}=r\left(\sinh \chi \cosh \chi \partial_{\chi}+i p \sinh ^{2} \chi+2\right) \mathcal{B}
$$

The components of $H_{\mu \nu}^{(o) p \ell m}$ in the Milne coordinates are given by the same formula as given in the case of even parity, Eq. (4.4).

We consider the gauge transformation

$$
\tilde{H}_{\mu \nu}^{(o) p \ell m}=H_{\mu \nu}^{(o) p \ell m}+\zeta_{\mu ; \nu}+\zeta_{\nu ; \mu}
$$

so as to make $\tilde{H}_{\xi \mu}^{(o) p \ell m}=0$.

The condition $\tilde{H}_{\xi A}^{(o) p \ell m}=0$ gives the equation

$$
\partial_{\xi}\left(\hat{\sigma}_{A B} \zeta^{B}\right)=-\frac{\sinh \chi}{r^{2}} \mathcal{B} \mathcal{Y}_{A}
$$

and it is integrated to be

$$
\sigma_{A B} \zeta^{B}=\frac{r}{i p+1} \mathcal{B Y}_{A}
$$

The other components are calculated by substituting the above equation to the formulas (4.21), replacing $\rho^{\mu}$ with $\zeta^{\mu}$ and the suffix $(e)$ with $(o)$. Then it is straightforward to check the following equalities,

$$
\tilde{H}_{\chi A}^{p \ell m}=\mathcal{H}_{\chi A}^{p \ell m}, \quad \tilde{H}_{A B}^{p \ell m}=\mathcal{H}_{A B}^{p \ell m}
$$

\section{SACHS-WOLFE EFFECT IN THE MILNE AND MINKOWSKI UNIVERSES}

In this section we evaluate the temperature anisotropy in the Milne and Minkowski universes due to the socalled Sachs-Wolfe effect of gravitational wave perturbations [23]. We consider the case when gravitational wave perturbations are in the Minkowski vacuum state. Since the background spacetime is empty, there is nothing that physically determines the last scattering surface. Hence we need to specify it by hand. Here we assume that the last scattering surface is at $\xi=\xi_{l s}$ in the case of the Milne universe and at $t=t_{l s}$ in the case of the Minkowski universe, and that there is no intrinsic temperature fluctuations when photons are emitted from this surface. This choice of the last scattering surface is not gauge invariant. Thus to make the problem definite the gauge must be fixed.

First we consider the case of the Milne universe. We take the synchronous gauge with respect to the Milne coordinates. That is, we set the gauge condition $h_{\xi \mu}=0$. Since this is the gauge we adopted for describing the gravitational wave modes in the Milne universe, we are ready to calculate the Sachs-Wolfe effect now.

To calculate the Sachs-Wolfe effect in the Milne universe, it is convenient to introduce the conformally transformed spacetime,

$$
\begin{aligned}
d \check{s}^{2} & =\xi^{-2} d s^{2} \\
& =-d \eta^{2}+d \chi^{2}+\sinh ^{2} \chi d \Omega^{2} \\
& =: \check{g}_{\mu \nu} d x^{\mu} d x^{\nu},
\end{aligned}
$$

where $\xi=e^{\eta}$. The corresponding metric perturbations in this transformed spacetime are given by

$$
\check{h}_{\mu \nu} d x^{\mu} d x^{\nu}=\xi^{-2} h_{\mu \nu} d x^{\mu} d x^{\nu} .
$$

Then taking the position of an observer at $\chi=0, \eta=\eta_{\text {obs }}$, the temperature fluctuation caused by the Sachs-Wolfe effect is given by 23]. (See Eq. (5.15))

$$
\frac{\delta T}{T}(\Omega)=\frac{1}{2} \int_{0}^{\eta_{o b s}-\eta_{l s}} d \lambda\left(\frac{\partial \check{h}_{\chi \chi}}{\partial \eta}\right)(\eta(\lambda), \chi(\lambda), \Omega),
$$

where

$$
\eta(\lambda)=\eta_{o b s}-\lambda, \quad \chi(\lambda)=\lambda .
$$

Since $\check{h}_{\chi \chi}=\xi^{-2} h_{\chi \chi}$ is the only component that causes the Sachs-Wolfe effect, only the even parity modes contribute to it. It is customary to describe the temperature anisotropy in terms of the multipole moments that are defined by 


$$
C(\gamma):=\left\langle 0\left|\frac{\delta T}{T}(\Omega) \frac{\delta T}{T}\left(\Omega^{\prime}\right)\right| 0\right\rangle=: \sum_{\ell=1}^{\infty} \frac{(2 \ell+1)}{4 \pi}\left\langle a_{\ell}^{2}\right\rangle P_{\ell}(\cos \gamma)
$$

where $\gamma$ is the angle between $\Omega$ and $\Omega^{\prime}$. By using the expression (3.36) for $\hat{h}_{\mu \nu}^{(e)}$ with the mode functions given by Eq. (3.42), $\left\langle a_{\ell}^{2}\right\rangle$ is calculated to be

$$
\begin{gathered}
\left\langle a_{\ell}^{2}\right\rangle=\int_{-\infty}^{\infty} d p \int_{0}^{\eta_{o b s}-\eta_{l s}} d \lambda \int_{0}^{\eta_{o b s}-\eta_{l s}} d \lambda^{\prime} \frac{(\ell-1) \ell(\ell+1)(\ell+2)}{32 \pi p^{2}} e^{\pi p}|\Gamma(i p+\ell+1)|^{2} \\
\times e^{-i p\left(\lambda-\lambda^{\prime}\right)} e^{-2 \eta_{o b s}+\lambda+\lambda^{\prime}} \frac{P_{i p-\frac{1}{2}}^{-\ell-\frac{1}{2}}(\cosh \lambda)}{(\sinh \lambda)^{5 / 2}} \frac{P_{i p-\frac{1}{2}}^{-\ell-\frac{1}{2}}\left(\cosh \lambda^{\prime}\right)}{\left(\sinh \lambda^{\prime}\right)^{5 / 2}} .
\end{gathered}
$$

Near $p=0$, the integrand behaves as $\sim 1 / p^{2}$, and so the $p$-integration in (5.6) is infrared divergent.f

Second, we consider the case of the Minkowski universe. Choosing the synchronous gauge $h_{t \mu}=0$, the temperature anisotropy is expressed as

$$
\frac{\delta T}{T}(\Omega)=\frac{1}{2} \int_{0}^{t_{o b s}-t_{l s}} d \lambda\left(\frac{\partial h_{r r}}{\partial t}\right)(t(\lambda), r(\lambda), \Omega)
$$

where

$$
t(\lambda)=t_{o b s}-\lambda, \quad r(\lambda)=\lambda
$$

By using the expression (3.15) for $\hat{h}_{\mu \nu}^{(e)}$, we find that $\left\langle a_{\ell}^{2}\right\rangle$ for the present case becomes

$$
\left\langle a_{\ell}^{2}\right\rangle=\int_{0}^{\infty} d k \int_{0}^{t_{o b s}-t_{l s}} d \lambda \int_{0}^{t_{o b s}-t_{l s}} d \lambda^{\prime} \frac{(\ell-1) \ell(\ell+1)(\ell+2)}{8 \pi k \lambda^{2} \lambda^{\prime 2}} e^{-i k\left(\lambda-\lambda^{\prime}\right)} j_{\ell}(k \lambda) j_{\ell}\left(k \lambda^{\prime}\right) .
$$

Different from Eq. (5.6), this k-integration does not diverge around $k=0$. 3

Thus we have a seemingly paradoxical result. The rms value of the temperature anisotropy diverges when calculated in the Milne coordinates while it converges when calculated in the Minkowski coordinates. Since we have already shown the equivalence of the Milne mode functions and the Minkowski mode functions, the only possible origin of this difference is the difference in the definition of the last scattering surfaces in the two models. Let us therefore investigate it in detail.

For this purpose, we reconsider the meaning of the Sachs-Wolfe formula. We begin with the perturbed geodesic equation expressed in terms of the Milne coordinates, in the conformally transformed spacetime,

$$
k^{\mu} \check{\nabla}_{\mu} k^{\nu}=-\Gamma_{(1) \sigma \rho}^{\nu} k^{\sigma} k^{\rho}
$$

where

$$
\Gamma_{(1) \sigma \rho}^{\nu}=\frac{1}{2} \check{g}^{\nu \mu}\left(\check{\nabla}_{\rho} \check{h}_{\mu \sigma}+\check{\nabla}_{\sigma} \check{h}_{\mu \rho}-\check{\nabla}_{\mu} \check{h}_{\rho \sigma}\right),
$$

and $\check{\nabla}_{\mu}$ is the covariant derivative with respect to the background metric in the conformally transformed spacetime. We expand $k^{\nu}$ as

$$
k^{\nu}=k_{(0)}^{\nu}+k_{(1)}^{\nu}
$$

where

$$
k_{(0)}^{\nu}=(-1,1,0,0),
$$

Then the geodesic equation is expanded as

\footnotetext{
${ }^{\ddagger}$ This divergence was first pointed out by B. Allen and R. Caldwell 19.

$\S$ This expression is found to be ultraviolet divergent after the $\lambda$-integrations [19]. However it should be distinguished from the infrared divergence in Eq. (5.6) because the present ultraviolet divergence can be removed by introducing a small cutoff at the lower limit of the $\lambda$-integrations. Of course, the equivalent divergence exists in the expression (5.6).
} 


$$
k_{(0)}^{\nu} \check{\nabla}_{\nu} k_{(1)}^{\mu}+k_{(1)}^{\nu} \check{\nabla}_{\nu} k_{(0)}^{\mu}=-\Gamma_{(1) \sigma \rho}^{\nu} k_{(0)}^{\sigma} k_{(0)}^{\rho}
$$

It gives

$$
\Delta k_{(1)}^{\eta}:=k_{(1)}^{\eta}\left(\lambda=\eta_{o b s}-\eta_{l s}\right)-k_{(1)}^{\eta}(\lambda=0)=\frac{1}{2} \int_{0}^{\eta_{o b s}-\eta_{s}} d \lambda\left(\partial_{\rho} \check{h}_{\sigma \eta}+\partial_{\sigma} \check{h}_{\rho \eta}-\partial_{\eta} \check{h}_{\sigma \rho}\right) k_{(0)}^{\sigma} k_{(0)}^{\rho} .
$$

In the synchronous gauge the above equation reduces to the standard formula (5.3). Under a gauge transformation given by

$$
\delta \check{h}_{\mu \nu}=2 \check{\nabla}_{(\mu} \check{\rho}_{\nu)},
$$

the component $\Delta k_{(1)}^{\eta}$ changes by

$$
\left.\left(\frac{d}{d \lambda} \check{\rho}_{\eta}\right)\right|_{0} ^{\eta_{o b s}-\eta_{l s}}
$$

Hence $\delta T / T$ is not invariant under a gauge transformation. This is not a surprise since this change is due to a shift of the definition of the last scattering surface. Before the gauge transformation the last scattering surface is at $\eta=\eta_{l s}$ but after the gauge transformation it is at $\tilde{\eta}=\eta-\check{\rho}^{\eta}=\eta_{l s}$.

Now it is easy to see that the origin of the divergence is this change of the last scattering surface. Observing Eq. (4.9), $\mathcal{A}$ is finite at $p \rightarrow 0$. Hence so is $H_{\mu \nu}^{(e) p \ell m}$. So if $\Delta k_{(1)}^{\eta}$ is evaluated by substituting $H_{\mu \nu}^{(e) p \ell m}$ defined by Eq. (4.3) into the formula (5.15), there will be no infrared divergence. This situation corresponds to the case when the last scattering surface is defined in the Minkowski synchronous gauge.

After the gauge transformation, (4.14), the mode functions become the Milne synchronous ones, $\mathcal{H}_{\mu \nu}^{(e) p \ell m}=\tilde{H}_{\mu \nu}^{(e) p \ell m}$. It was seen in Eq. (5.6) that if $\mathcal{H}_{\mu \nu}^{(e) p \ell m}$ is substituted into (5.15), the expression becomes infrared divergent. As explained above, the difference between these two is just caused by the difference in the definition of the last scattering surface, whose effect on $\Delta T / T$ is given by (5.17). It is easy to understand this contribution causes the infrared divergence if we notice the appearance of an additional singular factor of $p^{-1}$ in $\check{\rho}^{\eta}=\xi^{-1} \rho^{\xi}$ (See Eq.(4.16)).

We suspect a similar divergence to occur in the de Sitter universe if the open chart is used to evaluate the temperature anisotropy [19]. Then, does this divergence sign a crisis of theoretical framework? We claim that it is not the case but the divergence is solely due to the unphysical situation we have considered here. In the present model, the last scattering surface is defined by hand. So it does not have gauge invariant meaning. To make the problem physically gauge invariant, we need to include in the model some scalar quantity which determines the time slice of constant temperature in the universe. Then such a quantity will be inevitably coupled with gravity and background spacetime will be no longer Minkowskian (nor purely de Sitter). Hence the vacuum will be no longer highly symmetric as the Minkowski vacuum. Namely, if the time slicing defines a homogeneous and isotropic open universe, the symmetry of the vacuum will be $O(3,1)$ but not as symmetric as the Minkowski (or de Sitter) vacuum. We expect this 'symmetry breaking' will altar the power spectrum of gravitational waves and remove the divergence. In particular, in a realistic model of the open inflationary universe, we expect the rms value of the temperature anisotropy to be finite.

\section{SUMMARY}

In this paper, we considered the quantized gravitational waves in the Milne universe. We first constructed positive frequency functions of gravitational wave perturbations corresponding to the Minkowski vacuum state by means of the coordinates of the Milne universe. We used the analyticity of mode functions in the lower half of complex $t$-plane as a guiding principle to determine the positive frequency functions. In this process, we had to fix the normalization of the mode functions, which are to be determined by setting the appropriate commutation relations on a Cauchy surface. The Milne universe does not contain a Cauchy surface but its extension, i.e., the Rindler universe, does. Following the standard reduction scheme for the constrained system, we wrote down the reduced action for the physical degrees of freedom in the Rindler universe. Using this expression, quantization was performed and the normalization of the modes were determined.

Next, we examined the equivalence of the positive frequency functions of gravitational wave perturbations written in terms of the Minkowski coordinates and those in terms of the Milne coordinates. It was shown explicitly that they are related with each other by a unitary transformation and a succeeding gauge transformation.

Finally, we discussed the Sachs-Wolfe effect in the Milne universe. The contribution to temperature fluctuations in the Minkowski universe from low frequency modes does not have any bad behavior. However, a naive application of the Sachs-Wolfe formula to the Milne universe results in infrared divergent temperature fluctuations although the state is set in the Minkowski vacuum one. We clarified the origin of this divergence. In the Sachs-Wolfe formula for gravitational wave perturbations, the last scattering surface is chosen to be the time constant surface because the intrinsic fluctuations of the last scattering surface are to be attributed to scalar perturbations. However, this choice 
of the last scattering surface is not invariant under a gauge transformation if scalar perturbations are neglected. So the gauge transformation that was necessary to relate the mode functions in the Minkowski universe and in the Milne universe is the origin of this divergence.

We expect, however, that this divergence will be removed in a realistic model of an open inflationary universe. Further discussion on this issue will be given in a separate paper.

\section{Acknowledgments}

We thank B. Allen and R. Caldwell for insight into the problem of the divergence of the temperature fluctuation in the Milne universe and for useful comments and discussions. We also thank Y. Mino, and J. Soda for helpful discussions. This work was supported in part by Monbusho Grant-in-Aid for Scientific Research No.07304033.

\section{APPENDIX A: CANONICAL QUANTIZATION OF GRAVITATIONAL WAVES IN THE RINDLER UNIVERSE}

Here, we discuss the quantization of gravitational waves in the Rindler universe following the standard method to reduce the degrees of freedom of a constrained system to a physical ones. In this appendix the subscript $R$ in $\xi_{R}$ and $\chi_{R}$ is omitted for notational simplicity because the Milne coordinates will not be used.

We recapitulate the Lagrangian for the gravitational perturbation,

$$
L^{(2)}=\frac{1}{2}\left(-h_{\mu \nu ; \rho} h^{\mu \nu ; \rho}+2 h_{\mu \nu ; \rho} h^{\rho \mu ; \nu}-2 h_{\mu \nu}{ }^{\nu} h^{; \mu}+h_{; \mu} h^{; \mu}\right) .
$$

For later convenience, we introduce the unit normal vectors,

$$
\xi^{\mu}:=\left(\partial_{\xi}\right)^{\mu}=(1,0,0,0), \quad n^{\mu}:=\xi^{-1}\left(\partial_{\chi}\right)^{\mu}=\left(0, \xi^{-1}, 0,0\right) .
$$

Thus

$$
\eta_{\mu \nu}=\xi_{\mu} \xi_{\nu}-n_{\mu} n_{\nu}+\sigma_{\mu \nu}
$$

Further we adopt the convention to denote the projection of tensors as

$$
\begin{aligned}
& f_{\xi}:=f_{\mu} \xi^{\mu} \\
& f_{n}:=f_{\mu} n^{\mu}=\xi^{-1} f_{\chi} .
\end{aligned}
$$

The following relation is used in the following calculations.

$$
\begin{aligned}
n_{; \nu}^{\mu} & =-\frac{1}{\xi} \xi^{\mu} n_{\nu}+\frac{\tanh \chi}{\xi} \sigma_{\nu}^{\mu}, \\
\xi_{; \nu}^{\mu} & =-\frac{1}{\xi} n^{\mu} n_{\nu}+\frac{1}{\xi} \sigma_{\nu}^{\mu} .
\end{aligned}
$$

The each component of covariant derivatives of metric perturbations becomes

$$
\begin{aligned}
h_{n n ; n} & =\frac{1}{\xi} \partial_{\chi} h_{n n}-\frac{2}{\xi} h_{n \xi}, \\
h_{n n ; \xi} & =\partial_{\xi} h_{n n}, \\
h_{n n ; A} & =h_{n n \| A}-\frac{2 \tanh \chi}{\xi} h_{n A}, \\
h_{n \xi ; n} & =\frac{1}{\xi} \partial_{\chi} h_{n \xi}-\frac{1}{\xi}\left(h_{\xi \xi}+h_{n n}\right), \\
h_{n \xi ; \xi} & =\partial_{\xi} h_{n \xi}, \\
h_{n \xi ; A} & =h_{n \xi \| A}-\frac{\tanh \chi}{\xi} h_{\xi A}-\frac{1}{\xi} h_{n A}, \\
h_{\xi \xi ; n} & =\frac{1}{\xi} \partial_{\chi} h_{\xi \xi}-\frac{2}{\xi} h_{n \xi}, \\
h_{\xi \xi ; \xi} & =\partial_{\xi} h_{\xi \xi},
\end{aligned}
$$




$$
\begin{aligned}
h_{\xi \xi ; A} & =h_{\xi \xi \| A}-\frac{2}{\xi} h_{\xi A}, \\
h_{n A ; n} & =\frac{1}{\xi}\left(\partial_{\chi}-\tanh \chi\right) h_{n A}-\frac{1}{\xi} h_{\xi A}, \\
h_{n A ; \xi} & =\left(\partial_{\xi}-\frac{1}{\xi}\right) h_{n A}, \\
h_{n A ; B} & =h_{n A \| B}+\left(\frac{1}{\xi} h_{n \xi}-\frac{\tanh \chi}{\xi} h_{n n}\right) \sigma_{A B}-\frac{\tanh \chi}{\xi} h_{A B}, \\
h_{\xi A ; n} & =\frac{1}{\xi}\left(\partial_{\chi}-\tanh \chi\right) h_{\xi A}-\frac{1}{\xi} h_{n A}, \\
h_{\xi A ; \xi} & =\left(\partial_{\xi}-\frac{1}{\xi}\right) h_{\xi A}, \\
h_{\xi A ; B} & =h_{\xi A \| B}+\left(\frac{1}{\xi} h_{\xi \xi}-\frac{\tanh \chi}{\xi} h_{n \xi}\right) \sigma_{A B}-\frac{1}{\xi} h_{A B}, \\
h_{A B ; n} & =\frac{1}{\xi}\left(\partial_{\chi}-2 \tanh \chi\right) h_{A B}, \\
h_{A B ; \xi} & =\left(\partial_{\xi}-\frac{2}{\xi}\right) h_{A B}, \\
h_{A B ; C} & =h_{A B \| C}+\left(\frac{2}{\xi} h_{\xi(A} \sigma_{B) C}-\frac{2 \tanh \chi}{\xi} h_{n(A} \sigma_{B) C}\right),
\end{aligned}
$$

where we used the abbreviated notation such as $h_{n A ; \xi} \equiv h_{\mu \nu ; \rho} n^{\mu} \sigma_{A}^{\nu} \xi^{\rho}$. Below we expand the metric perturbation in terms of the spherical harmonics and consider the even and odd parity modes separately.

\section{1. even parity}

Concentrating on the even parity modes, we expand the variables by using the spherical harmonics $Y=Y_{\ell m}(\Omega)$,

$$
\begin{aligned}
& h_{n n}^{(e)}=\sum H_{n n}^{(e) \ell m} Y, \quad h_{n \xi}^{(e)}=\sum H_{n \xi}^{(e) \ell m} Y, \quad h_{\xi \xi}^{(e)}=\sum H_{\xi \xi}^{(e) \ell m} Y, \\
& h_{n A}^{(e)}=\sum H_{n}^{(e) \ell m} Y_{\| A}, \quad h_{\xi A}^{(e)}=\sum H_{\xi}^{(e) \ell m} Y_{\| A}, \\
& h_{A B}^{(e)}=\sum\left(w^{(e) \ell m} Y \hat{\sigma}_{A B}+v^{(e) \ell m} Y_{A B}\right)
\end{aligned}
$$

where

$$
Y_{A B}=\frac{Y_{\| A B}}{\ell(\ell+1)}+\frac{1}{2} \hat{\sigma}_{A B} Y
$$

The reality condition implies $\overline{H_{i}^{\ell m}}=H_{i}^{\ell-m}$, where $H_{i}=H_{n n}, H_{n \xi}, H_{n}, H_{\xi \xi}, H_{\xi}, w, v$. To keep the simplicity of notation, we often abbreviate the indices, $(e), \ell$ and $m$, unless there arises confusion.

For later convenience, we list the formulas of the $\Omega$-integration,

$$
\begin{aligned}
\int d \Omega Y \bar{Y} & =1, \\
\int d \Omega \hat{\sigma}^{A A^{\prime}} Y_{\| A} \overline{Y_{\| A^{\prime}}} & =\ell(\ell+1), \\
\int d \Omega \hat{\sigma}^{A A^{\prime}} \hat{\sigma}^{B B^{\prime}} Y_{A B} \overline{Y_{A^{\prime} B^{\prime}}} & =\frac{\ell(\ell+1)-2}{2 \ell(\ell+1)}, \\
\int d \Omega \hat{\sigma}^{A A^{\prime}} \hat{\sigma}^{B B^{\prime}} \hat{\sigma}^{C C^{\prime}} Y_{A B \| C} \overline{Y_{A^{\prime} B^{\prime} \| C^{\prime}}} & =\frac{(\ell(\ell+1)-2)(\ell(\ell+1)-4)}{2 \ell(\ell+1)}, \\
\int d \Omega \hat{\sigma}^{A A^{\prime}} \hat{\sigma}^{B B^{\prime}} \hat{\sigma}^{C C^{\prime}} Y_{A B \| C} \overline{Y_{A^{\prime} C^{\prime} \| B^{\prime}}} & =\frac{(\ell(\ell+1)-2)(\ell(\ell+1)-6)}{4 \ell(\ell+1)} .
\end{aligned}
$$

It is convenient to rewrite the components having more than two of their indices projected onto $\Omega$-sphere;

$$
h_{n A ; B}=\left[-\frac{\ell(\ell+1)}{2 \xi^{2} \cosh ^{2} \chi} H_{n}+\frac{1}{\xi} H_{n \xi}-\frac{\tanh \chi}{\xi}\left(H_{n n}+\frac{w}{\xi^{2} \cosh ^{2} \chi}\right)\right] \sigma_{A B} Y
$$




$$
\begin{aligned}
& +\left[\ell(\ell+1) H_{n}-\frac{\tanh \chi}{\xi} v\right] Y_{A B}, \\
h_{\xi A ; B}=[ & \left.-\frac{\ell(\ell+1)}{2 \xi^{2} \cosh ^{2} \chi} H_{\xi}+\frac{1}{\xi}\left(H_{\xi \xi}-\frac{w}{\xi^{2} \cosh ^{2} \chi}\right)-\frac{\tanh \chi}{\xi} H_{n \xi}\right] \sigma_{A B} Y \\
& +\left[\ell(\ell+1) H_{\xi}-\frac{1}{\xi} v\right] Y_{A B}, \\
h_{A B ; n}= & \frac{\sigma_{A B} Y}{\xi^{3} \cosh ^{2} \chi}\left[\partial_{\chi}-2 \tanh \chi\right] w+\frac{1}{\xi} Y_{A B}\left[\partial_{\chi}-2 \tanh \chi\right] v, \\
h_{A B ; \xi}= & \frac{\sigma_{A B} Y}{\xi^{2} \cosh ^{2} \chi}\left[\partial_{\xi}-\frac{2}{\xi}\right] w+Y_{A B}\left[\partial_{\xi}-\frac{2}{\xi}\right] v, \\
h_{A B ; C}= & \frac{w}{\xi^{2} \cosh ^{2} \chi} Y_{\| C} \sigma_{A B}+v Y_{A B \| C}+\frac{2}{\xi}\left[H_{\xi}-\tanh \chi H_{n}\right] Y_{\|(A} \sigma_{B) C} .
\end{aligned}
$$

Then it is straightforward to calculate the Lagrangian for the even parity modes by substituting (A7) into (A1). By using the formulas (A9) the $\Omega$-integration in the action is performed:

$$
\int d^{4} x \sqrt{-g} L^{(2)}\left[h_{\mu \nu}^{(e)}\right]=\int d \chi \int d \xi \mathbf{L}^{(e)} .
$$

Here we demonstrate the most complicated terms;

$$
\begin{aligned}
& \int d \Omega \sigma^{\mu \mu^{\prime}} \sigma^{\nu \nu^{\prime}} \sigma^{\rho \rho^{\prime}}\left(-h_{\mu \nu ; \rho} h_{\mu^{\prime} \nu^{\prime} ; \rho^{\prime}}+2 h_{\mu \nu ; \rho} h_{\nu^{\prime} \rho^{\prime} ; \mu^{\prime}}\right) \\
& =\sum_{\ell, m} \frac{1}{\left(\xi^{2} \cosh ^{2} \chi\right)^{3}}\left[-\frac{\ell(\ell+1)-2}{\ell(\ell+1)}|v|^{2}+4 \ell(\ell+1)\left(\xi \cosh ^{2} \chi\right)^{2}\left|H_{\xi}-\tanh \chi H_{n}\right|^{2}\right. \\
& \left.\quad+8 \ell(\ell+1) \xi \cosh ^{2} \chi\left[H_{\xi}-\tanh \chi H_{n}\right] \bar{w}-2(\ell(\ell+1)-2) v \bar{w}\right] .
\end{aligned}
$$

Next we define the canonical conjugate momentum by

$$
\overline{P_{i}^{(e) \ell m}}:=\frac{\partial \mathbf{L}^{(e)}}{\partial\left(\partial_{\chi} H_{i}^{(e) \ell m}\right)},
$$

where $P_{i}=P_{n n}, P_{n \xi}, P_{n}, P_{\xi \xi}, P_{\xi}, P_{w}, P_{v}$. Since the $\chi$-derivatives of $H_{n n}, H_{n \xi}$ and $H_{n}$ are not contained in the defining equations of the conjugate momenta, they give the constraint equations

$$
\begin{aligned}
& C_{1}:=P_{n n}-\ell(\ell+1) H_{n}-2 \xi \cosh \chi \sinh \chi H_{n n}-\frac{2 \tanh \chi}{\xi} w+\xi^{2} \cosh ^{2} \chi\left[\partial_{\xi}+\frac{2}{\xi}\right] H_{n \xi}=0, \\
& C_{2}:=P_{n \xi}-\xi^{2} \cosh ^{2} \chi \partial_{\xi}\left(H_{n n}+H_{\xi \xi}\right)-2\left[\partial_{\xi}-\frac{2}{\xi}\right] w=0, \\
& C_{3}:=P_{n}-\ell(\ell+1)\left(H_{n n}+H_{\xi \xi}+\frac{2}{\xi^{2} \cosh ^{2} \chi} w-\frac{4 \tanh \chi}{\xi} H_{n}\right)=0 .
\end{aligned}
$$

The other components are

$$
\begin{aligned}
P_{\xi \xi} & =-\frac{2}{\xi} \partial_{\chi} w-\ell(\ell+1) H_{n}-2 \xi \cosh \chi \sinh \chi H_{n n}+\frac{2 \tanh \chi}{\xi} w-\xi^{2} \cosh ^{2} \chi\left[\partial_{\xi}-\frac{2}{\xi}\right] H_{n \xi}, \\
P_{\xi} & =2 \ell(\ell+1)\left(\frac{1}{\xi} \partial_{\chi} H_{\xi}-H_{n \xi}-\left[\partial_{\xi}-\frac{1}{\xi}\right] H_{n}\right), \\
P_{w} & =-\frac{2}{\xi^{3} \cosh ^{2} \chi}\left[\partial_{\chi}-2 \tanh \chi\right] w-\frac{2}{\xi} \partial_{\chi} H_{\xi \xi}+2\left[\partial_{\xi}+\frac{2}{\xi}\right] H_{n \xi}, \\
P_{v} & =\frac{\ell(\ell+1)-2}{2 \ell(\ell+1)} \frac{1}{\xi^{3} \cosh ^{2} \chi} \partial_{\chi} v-\frac{\ell(\ell+1)-2}{\xi^{2} \cosh ^{2} \chi} H_{n} .
\end{aligned}
$$

The Hamiltonian is defined by

$$
\mathbf{H}^{(e)}=\sum_{\ell, m} \sum_{i} \overline{P_{i}^{(e)}}\left(\partial_{\chi} H_{i}^{(e)}\right)-\mathbf{L}^{(e)},
$$


where $\partial_{\chi} H_{n n}, \partial_{\chi} H_{n \xi}$ and $\partial_{\chi} H_{n}$ are to be replaced by $\lambda_{1}, \lambda_{2}$ and $\lambda_{3}$, respectively.

The canonical equations of motion are

$$
\begin{aligned}
H_{i} & =\frac{\partial \mathbf{H}}{\partial \overline{P_{i}}}, \\
P_{i} & =-\frac{\partial \mathbf{H}}{\partial \overline{H_{i}}} .
\end{aligned}
$$

We set the gauge conditions

$$
H_{\xi \xi}=0, \quad H_{\xi}=0, \quad H_{n \xi}=0,
$$

in accordance with $h_{\xi \mu}=0$. These gauge conditions imply the consistency conditions $\quad \partial_{\chi} H_{\xi \xi}=0, \quad \partial_{\chi} H_{\xi}=0$ and $\partial_{\chi} H_{n \xi}=0$, which become

$$
\begin{aligned}
0=D_{1} & =P_{\xi \xi}+\ell(\ell+1) H_{n}+2 \xi \sinh \chi \cosh \chi H_{n n}+\frac{2}{\xi} \tanh \chi w-\xi^{2} \cosh ^{2} \chi P_{w}, \\
0=D_{2} & =P_{\xi}+2 \ell(\ell+1)\left(\partial_{\xi}-\frac{1}{\xi}\right) H_{n}, \\
\lambda_{2} & =0
\end{aligned}
$$

respectively. Before going further to examine the consistency conditions for the gauge conditions, we consider the consistency condition for the constraint equations. From $\partial_{\chi} C_{1}=0$ and $\partial_{\chi} C_{2}=0$, we obtain

$$
\begin{aligned}
& H_{n}=\frac{\operatorname{coth} \chi}{\ell(\ell+1) \xi}\left[\left(2-2 \hat{K}-\frac{\ell(\ell+1)}{\cosh ^{2} \chi}\right) w-\frac{\ell(\ell+1)-2}{2 \cosh ^{2} \chi} v\right], \\
& P_{w}=\frac{1}{\xi^{3} \sinh \chi \cosh \chi}\left[2 \xi^{2} \sinh ^{2} \chi H_{n n}+\left(4-2 \hat{K}-\frac{\ell(\ell+1)+2}{\cosh ^{2} \chi}\right) w-\frac{\ell(\ell+1)-2}{2 \cosh ^{2} \chi} v\right],
\end{aligned}
$$

and from $\partial_{\chi} C_{3}=0$ we obtain

$$
P_{v}=\frac{\operatorname{coth} \chi}{2 \ell(\ell+1) \xi^{3}}\left[\left(-4 \hat{K}(\hat{K}-1)+\frac{\ell(\ell+1)(\ell(\ell+1)-2)}{\cosh ^{4} \chi}\right) w+(\ell(\ell+1)-2)\left(2-\hat{K}+\frac{\ell(\ell+1)-4}{2 \cosh ^{2} \chi}\right) \frac{v}{\cosh ^{2} \chi}\right] .
$$

Here we introduced the derivative operator

$$
\hat{K}=-\xi^{3} \partial_{\xi} \xi^{-1} \partial_{\xi}
$$

Using the relations which have been already obtained, the second level consistency conditions for the gauge conditions, $\partial_{\chi} D_{1}=0$ and $\partial_{\chi} D_{2}=0$ reduce to

$$
E_{1}:=H_{n n}-\frac{2 w}{\xi^{2} \cosh ^{2} \chi}=0
$$

and

$$
\lambda_{3}=\frac{1}{\ell(\ell+1) \xi}\left[\left(4(\hat{K}-1)+\frac{3 \ell(\ell+1)}{\cosh ^{2} \chi}\right) w+\frac{\ell(\ell+1)-2}{2 \cosh ^{2} \chi} v\right],
$$

respectively. Furthermore $\partial_{\chi} E_{1}=0$ gives the condition

$$
\lambda_{1}=\frac{2}{\xi^{2} \cosh \chi \sinh \chi}\left[\left((\hat{K}-4)+\frac{\ell(\ell+1)+6}{2 \cosh ^{2} \chi}\right) w+\frac{\ell(\ell+1)-2}{4 \cosh ^{2} \chi} v\right] .
$$

Now only the second level consistency conditions for the three constraint equations, $\partial_{\chi}^{2} C_{i}=0$, are remaining. These conditions are found to be satisfied by using the relations which we have already obtained and so they do not give any new condition. Thus we found the system of the primary and the secondary constraints closes.

Now we find that the equation for $w$ reduces to

$$
\partial_{\chi} w=\frac{\Pi}{\cosh ^{2} \chi}
$$




$$
\partial_{\chi} \Pi=\left[-\ell(\ell+1)-\hat{K} \cosh ^{2} \chi\right] w
$$

where $\Pi$ is defined by

$$
\Pi^{(e) \ell m}:=-\cosh ^{2} \chi\left[\frac{\ell(\ell+1) \xi}{2} H_{n}^{(e) \ell m}+\tanh \chi w^{(e) \ell m}\right] .
$$

All the other variables can be written in terms of $w$ and $\Pi$ as

$$
\begin{aligned}
H_{n n} & =\frac{2 w}{\xi^{2} \cosh ^{2} \chi}, \\
H_{n} & =-\frac{2}{\ell(\ell+1) \xi}\left[\tanh \chi w+\frac{\Pi}{\cosh ^{2} \chi}\right], \\
H_{\xi} & =H_{\xi \xi}=H_{n \xi}=0, \\
v & =\frac{4 \cosh ^{2} \chi}{\ell(\ell+1)-2}\left[\left(2-\hat{K}-\frac{\ell(\ell+1)+2}{2 \cosh ^{2} \chi}\right) w+\frac{\tanh \chi}{\cosh ^{2} \chi} \Pi\right], \\
P_{n n} & =\frac{2}{\xi}\left[2 \tanh \chi w-\frac{\Pi}{\cosh ^{2} \chi}\right], \\
P_{n} & =\frac{8}{\xi^{2}}\left[\left(1+\frac{\ell(\ell+1)-2}{2 \cosh ^{2} \chi}\right) w+\frac{\tanh ^{2} \chi}{\cosh ^{2} \chi} \Pi\right], \\
P_{n \xi} & =4\left(\partial_{\xi}-\frac{2}{\xi}\right) w, \\
P_{\xi \xi} & =0, \\
P_{\xi} & =\frac{4}{\xi}\left(\partial_{\xi}-\frac{2}{\xi}\right)\left(\tanh \chi w+\frac{\Pi}{\cosh ^{2} \chi}\right), \\
P_{w} & =\frac{2}{\xi^{3} \cosh ^{2} \chi}\left(2 \tanh \chi w-\frac{\Pi}{\cosh ^{2} \chi}\right), \\
P_{v} & =\frac{2}{\ell(\ell+1) \xi^{3}}\left[\left(4-3 \hat{K}-\frac{2}{\cosh ^{2} \chi}\right) \tanh \chi w+\left(2-\hat{K}+\frac{\ell(\ell+1)-4}{2 \cosh ^{2} \chi}\right) \frac{\Pi}{\cosh ^{2} \chi}\right] .
\end{aligned}
$$

Of course, Eqs. (A26) and (A28) are consistent with the mode functions obtained in Section 3.

Substituting (A28) into the canonical form of the action

$$
\int d \chi \int d \xi \mathcal{L}^{(e) \ell m}:=\int d \chi \int d \xi\left(\sum_{\ell, m} \sum_{i} \overline{P_{i}^{(e) \ell m}}\left(\partial_{\chi} H_{i}^{(e) \ell m}\right)-\mathbf{H}^{(e)}\right)
$$

the reduced action becomes

$$
\begin{aligned}
\int d \chi \int d \xi \mathcal{L}_{(r e d)}^{(e)} & =\sum_{\ell, m} \frac{8}{(\ell-1) \ell(\ell+1)(\ell+2)} \int d \chi \int \frac{d \xi}{\xi^{3}} \\
& \times\left[\bar{\Pi} \hat{K}(\hat{K}-1)\left(\partial_{\chi} w\right)-\frac{1}{2}\left(\frac{1}{\cosh ^{2} \chi} \bar{\Pi} \hat{K}(\hat{K}-1) \Pi+\bar{w} \hat{K}(\hat{K}-1)\left\{\ell(\ell+1)+\hat{K} \cosh ^{2} \chi\right\} w\right)\right] .
\end{aligned}
$$

Then we can see easily that $w$ and $\Pi$ can be expanded by using the eigen function of the operator $\hat{K}$. The normalized eigen functions should satisfy

$$
\hat{K} f_{p}=\left(p^{2}+1\right) f_{p}
$$

and

$$
\int_{0}^{\infty} \frac{d \xi}{\xi^{3}} f_{p} f_{p^{\prime}}=\delta\left(p-p^{\prime}\right)
$$

Thus we find $f_{p}=-\xi^{-i p+1} / \sqrt{2 \pi} \equiv-\xi^{2} \mathcal{U}_{p}$, where $\mathcal{U}_{p}$ is defined in Eq. (2.22). We expand the variables $w$ and $\Pi$ as

$$
w^{(e) \ell m}=-\xi^{2} \int d p w_{(e) p \ell m} \mathcal{U}_{p}, \quad \Pi^{(e) \ell m}=-\xi^{2} \int d p \Pi_{(e) p \ell m} \mathcal{U}_{p}
$$


Then the reality condition becomes

$$
\overline{w_{p \ell m}}=w_{-p \ell-m} \text {. }
$$

Using this expansion, the final form of the reduced action for the even parity modes becomes

$$
\begin{aligned}
\int d \chi d \xi \mathcal{L}_{(r e d)}^{(e)}= & \int_{-\infty}^{\infty} d p \sum_{\ell, m} \frac{8 p^{2}\left(p^{2}+1\right)}{(\ell-1) \ell(\ell+1)(\ell+2)} \\
& \times \int d \chi\left[\overline{\Pi_{(e) p \ell m}}\left(\partial_{\chi} w_{(e) p \ell m}\right)-\frac{1}{2}\left(\frac{\left|\Pi_{(e) p \ell m}\right|^{2}}{\cosh ^{2} \chi}+\left\{\ell(\ell+1)+\left(p^{2}+1\right) \cosh ^{2} \chi\right\}\left|w_{(e) p \ell m}\right|^{2}\right)\right] .
\end{aligned}
$$

Now we consider the quantization. We expand the operator $\hat{w}^{(e)}$, which is the quantum counter part of $w^{(e)}:=$ $\sum_{\ell, m} w^{(e) \ell m} Y_{\ell m}$, as

$$
\hat{w}^{(e)}=-\xi^{2} \int_{-\infty}^{\infty} d p \sum_{\ell, m}\left(w_{(e) p \ell m}(\chi) \mathcal{U}_{p}(\xi) Y_{\ell m}(\Omega) \hat{a}_{(e) p \ell m}+\text { h.c. }\right)
$$

Since the mode functions are already obtained in Eq. (3.42), comparison of the traceless part of $\mathcal{H}_{A B}^{(e) p \ell m}$ with the definition of $w$ in Eq. (A7) readily gives the solution for $w_{(e) p \ell m}(\chi)$,

$$
w_{(e) p \ell m}=\mathcal{N}_{(e) p \ell m}\left(\mathcal{T}_{4}^{p \ell}-\frac{\ell(\ell+1)}{2} \mathcal{T}_{3}^{p \ell}\right)=-\frac{\mathcal{N}_{(e) p \ell m}}{2} \mathcal{P}_{p \ell},
$$

which, of course, satisfies the equation of motion (A26). Then

$$
\begin{aligned}
\hat{w}^{(e)} & =\frac{\xi^{2}}{2} \int_{-\infty}^{\infty} d p \sum_{\ell, m}\left(\mathcal{N}_{(e) p \ell m} \mathcal{P}_{p \ell} \mathcal{U}_{p} Y_{\ell m} \hat{a}_{(e) p \ell m}+\text { h.c. }\right) \\
& =\frac{\xi^{2}}{2} \int_{-\infty}^{\infty} d p \sum_{\ell, m}\left(\mathcal{N}_{(e) p \ell m} \mathcal{P}_{p \ell} \hat{a}_{(e) p \ell m}+\frac{\Gamma(i p+\ell+1)}{\Gamma(-i p+\ell+1)} \overline{\mathcal{N}_{(e)-p \ell-m} \mathcal{P}_{p \ell}} \hat{a}_{(e)-p \ell-m}^{\dagger}\right) \mathcal{U}_{p} Y_{\ell m}
\end{aligned}
$$

where we used the relations which hold in the Rindler universe,

$$
\begin{aligned}
\overline{\mathcal{U}_{p}} & =\mathcal{U}_{-p}, \\
\overline{\mathcal{P}_{-p \ell}} & =\frac{\Gamma(i p+\ell+1)}{\Gamma(-i p+\ell+1)} \overline{\mathcal{P}_{p \ell}} .
\end{aligned}
$$

From this expression the quantum operator $\hat{w}_{(e) p \ell m}$ corresponding to $w_{(e) p \ell m}$ in the reduced action (A35) is read off

$$
\hat{w}_{(e) p \ell m}=-\frac{1}{2}\left(\mathcal{N}_{(e) p \ell m} \mathcal{P}_{p \ell} \hat{a}_{(e) p \ell m}+\frac{\Gamma(i p+\ell+1)}{\Gamma(-i p+\ell+1)} \overline{\mathcal{N}_{(e)-p \ell-m} \mathcal{P}_{p \ell}} \hat{a}_{(e)-p \ell-m}^{\dagger}\right)
$$

which, of course, satisfies the relation

$$
\hat{w}_{(e) p \ell m}^{\dagger}=\hat{w}_{(e)-p \ell-m}
$$

corresponding to the reality condition (A34).

With the aid of Eq. (A26), Eq. A35 determines the canonical commutation relations to be imposed,

$$
\begin{aligned}
& \frac{8 p^{2}\left(p^{2}+1\right) \cosh ^{2} \chi}{(\ell-1) \ell(\ell+1)(\ell+2)}\left[\hat{w}_{(e) p \ell m}, \partial_{\chi} \hat{w}_{(e) p^{\prime} \ell^{\prime} m^{\prime}}^{\dagger}\right]=i \delta\left(p-p^{\prime}\right) \delta_{\ell, \ell^{\prime}} \delta_{m, m^{\prime}}, \\
& {\left[\hat{w}_{(e) p \ell m}, \hat{w}_{(e) p^{\prime} \ell^{\prime} m^{\prime}}^{\dagger}\right]=0, \quad\left[\partial_{\chi} \hat{w}_{(e) p \ell m}, \partial_{\chi} \hat{w}_{(e) p^{\prime} \ell^{\prime} m^{\prime}}^{\dagger}\right]=0 .}
\end{aligned}
$$

Substituting ( $\mathrm{A} 40$ ) into the above relations, we finally obtain the result given in Eq. (3.43). 


\section{2. odd parity}

We recapitulate the odd parity 2-dimensional vector and tensor harmonics introduced in section 3 ,

$$
\mathcal{Y}_{A}:=Y_{\| C} \hat{\epsilon}_{A}^{C}, \quad \mathcal{Y}_{A B}:=Y_{\| C(A} \hat{\epsilon}_{B)}^{C}=\ell(\ell+1) Y_{C(A} \hat{\epsilon}_{B)}^{C},
$$

where $\hat{\epsilon}_{A B}$ is the unit antisymmetric tensor on the unit 2 -sphere with the metric $\hat{\sigma}_{A B}$. We note a basic relation between $\hat{\epsilon}_{A B}$ and $\hat{\sigma}_{A B}$,

$$
\hat{\epsilon}_{A B} \hat{\epsilon}_{C D}=\hat{\sigma}_{A C} \hat{\sigma}_{B D}-\hat{\sigma}_{A D} \hat{\sigma}_{B C} .
$$

We list the formulas,

$$
\begin{aligned}
\int d \Omega \hat{\sigma}^{A A^{\prime}} \mathcal{Y}_{A} \mathcal{Y}_{A^{\prime}} & =\ell(\ell+1), \\
\int d \Omega \hat{\sigma}^{A A^{\prime}} \hat{\sigma}^{B B^{\prime}} \mathcal{Y}_{A B} \mathcal{Y}_{A^{\prime} B^{\prime}} & =\frac{1}{2} \ell(\ell+1)(\ell(\ell+1)-2), \\
\int d \Omega \hat{\sigma}^{A A^{\prime}} \hat{\sigma}^{B B^{\prime}} \mathcal{Y}_{[A \| B]} \mathcal{Y}_{\left[A^{\prime} \| B^{\prime}\right]} & =\frac{1}{2} \ell^{2}(\ell+1)^{2}, \\
\int d \Omega \hat{\sigma}^{A A^{\prime}} \hat{\sigma}^{B B^{\prime}} \hat{\sigma}^{C C^{\prime}} \mathcal{Y}_{A B \| C} \mathcal{Y}_{A^{\prime} B^{\prime} \| C^{\prime}} & =\frac{1}{2} \ell(\ell+1)(\ell(\ell+1)-2)(\ell(\ell+1)-4), \\
\int d \Omega \hat{\sigma}^{A A^{\prime}} \hat{\sigma}^{B B^{\prime}} \hat{\sigma}^{C C^{\prime}} \mathcal{Y}_{A B \| C} \mathcal{Y}_{B^{\prime} C^{\prime} \| A^{\prime}} & =\frac{1}{4} \ell(\ell+1)(\ell(\ell+1)-2)(\ell(\ell+1)-6), \\
\int d \Omega \hat{\sigma}^{A A^{\prime}} \hat{\sigma}^{B C} \mathcal{Y}_{A B \| C} \mathcal{Y}_{A^{\prime}} & =-\ell(\ell+1)(\ell(\ell+1)-2) .
\end{aligned}
$$

We expand the metric perturbation in terms of the spherical harmonics as

$$
h_{n A}^{(o)}=\sum H_{n}^{(o) \ell m} \mathcal{Y}_{A}, \quad h_{\xi A}^{(o)}=\sum H_{\xi}^{(o) \ell m} \mathcal{Y}_{A}, \quad h_{A B}^{(o)}=\sum w^{(o) \ell m} \mathcal{Y}_{A B}
$$

The subscript $(o)$ is sometimes suppressed, too. Then we have

$$
\begin{aligned}
& h_{n n ; A}=-\frac{2 \tanh \chi}{\xi} H_{n} \mathcal{Y}_{A}, \\
& h_{\xi n ; A}=\left(-\frac{\tanh \chi}{\xi} H_{\xi}-\frac{1}{\xi} H_{n}\right) \mathcal{Y}_{A}, \\
& h_{\xi \xi ; A}=-\frac{2}{\xi} H_{\xi} \mathcal{Y}_{A}, \\
& h_{n A ; n}=\left(\frac{1}{\xi}\left(\partial_{\chi}-\tanh \chi\right) H_{n}-\frac{1}{\xi} H_{\xi}\right) \mathcal{Y}_{A} \text {, } \\
& h_{n A ; \xi}=\left(\partial_{\xi}-\frac{1}{\xi}\right) H_{n} \mathcal{Y}_{A}, \\
& h_{\xi A ; n}=\left(\frac{1}{\xi}\left(\partial_{\chi}-\tanh \chi\right) H_{\xi}-\frac{1}{\xi} H_{n}\right) \mathcal{Y}_{A} \text {, } \\
& h_{\xi A ; \xi}=\left(\partial_{\xi}-\frac{1}{\xi}\right) H_{\xi} \mathcal{Y}_{A}, \\
& h_{n[A ; B]}=H_{n} \mathcal{Y}_{[A \| B]} \text {, } \\
& h_{n(A ; B)}=\left(H_{n}-\frac{\tanh \chi}{\xi} w\right) \mathcal{Y}_{A B} \text {, } \\
& h_{\xi[A ; B]}=H_{\xi} \mathcal{Y}_{[A \| B]} \text {, } \\
& h_{\xi(A ; B)}=\left(H_{\xi}-\frac{1}{\xi} w\right) \mathcal{Y}_{A B} \text {, } \\
& h_{A B ; n}=\frac{1}{\xi}\left(\partial_{\chi}-2 \tanh \chi\right) w \mathcal{Y}_{A B}, \\
& h_{A B ; \xi}=\left(\partial_{\xi}-\frac{2}{\xi}\right) w \mathcal{Y}_{A B} \text {, } \\
& h_{A B ; C}=w \mathcal{Y}_{A B \| C}+\left(\frac{2}{\xi} H_{\xi}-\frac{2 \tanh \chi}{\xi} H_{n}\right) \mathcal{Y}_{\left({ }_{A} \sigma_{B) C}\right.} \text {. }
\end{aligned}
$$


Here we also used the abbreviated notation such as $h_{n A ; \xi} \equiv h_{\mu \nu ; \rho} n^{\mu} \sigma_{A}^{\nu} \xi^{\rho}$. The reality condition implies $\overline{H_{i}^{\ell m}}=H_{i}^{\ell-m}$. It will be worth noting that the Lagrangian for the odd parity modes are expressed as

$$
\begin{aligned}
L^{(2)}\left[h_{\mu \nu}^{(o)}\right]=\frac{1}{2} & \left\{h_{n A ; n} h_{n n ; A^{\prime}}-h_{n n ; A} h_{n n ; A^{\prime}}+4 h_{\xi A ; \xi} h_{\xi \xi ; A^{\prime}}-h_{\xi \xi ; A} h_{\xi \xi ; A^{\prime}}\right. \\
& +2\left(h_{n A ; \xi} h_{n A^{\prime} ; \xi}+h_{\xi A ; n} h_{\xi A^{\prime} ; n}+h_{n \xi ; A} h_{n \xi ; A^{\prime}}\right) \\
& \left.-4\left(h_{n A ; \xi} h_{n \xi ; A^{\prime}}+h_{\xi A ; n} h_{n \xi ; A^{\prime}}+h_{\xi A ; n} h_{n A^{\prime} ; \xi}\right)\right\} \sigma^{A A^{\prime}} \\
+ & \frac{1}{2}\left\{4 h_{n[A ; B]} h_{n\left[A^{\prime} ; B^{\prime}\right]}-4 h_{n(A ; B)} h_{n\left(A^{\prime} ; B^{\prime}\right)}+h_{A B ; n} h_{A^{\prime} B^{\prime} ; n}\right. \\
& \left.-4 h_{\xi[A ; B]} h_{\xi\left[A^{\prime} ; B^{\prime}\right]}+4 h_{\xi(A ; B)} h_{\xi\left(A^{\prime} ; B^{\prime}\right)}-h_{A B ; \xi} h_{A^{\prime} B^{\prime} ; \xi}\right\} \sigma^{A A^{\prime}} \sigma^{B B^{\prime}} \\
+ & \frac{1}{2}\left\{-h_{A B ; C} h_{A^{\prime} B^{\prime} ; C^{\prime}}+2 h_{A B ; C} h_{B^{\prime} C^{\prime} ; A^{\prime}}\right\} \sigma^{A A^{\prime}} \sigma^{B B^{\prime}} \sigma^{C C^{\prime}},
\end{aligned}
$$

where we used the fact that the odd parity modes are traceless by construction.

Then by using the formulas (A45), the $\Omega$-integration in the action is performed:

$$
\int d^{4} x \sqrt{-g} L^{(2)}\left[h_{\mu \nu}^{(o)}\right]=\int d \chi \int d \xi \mathbf{L}^{(o)} .
$$

Here we demonstrate the most complicated terms;

$$
\begin{aligned}
& \int d \Omega \sigma^{\mu \mu^{\prime}} \sigma^{\nu \nu^{\prime}} \sigma^{\rho \rho^{\prime}}\left(-h_{\mu \nu ; \rho} h_{\mu^{\prime} \nu^{\prime} ; \rho^{\prime}}+2 h_{\mu \nu ; \rho} h_{\nu^{\prime} \rho^{\prime} ; \mu^{\prime}}\right) \\
& \quad=\sum_{\ell, m} \frac{1}{\left(\xi^{2} \cosh ^{2} \chi\right)^{3}}\left[-\ell(\ell+1)(\ell(\ell+1)-2)|w|^{2}+4 \ell(\ell+1)\left(\xi \cosh ^{2} \chi\right)^{2}\left|H_{\xi}-\tanh \chi H_{n}\right|^{2}\right] .
\end{aligned}
$$

Next we define the canonical conjugate momentum by

$$
\overline{P_{i}^{(o) \ell m}}=\frac{\partial \mathbf{L}}{\partial\left(\partial_{\chi} H_{i}^{(o) \ell m}\right)}
$$

The defining equation of the conjugate momentum of $H_{n}$ gives the constraint equation

$$
C:=P_{n}+\frac{4 \ell(\ell+1)}{\xi} \tanh \chi H_{n}=0 .
$$

The other components are

$$
\begin{aligned}
P_{\xi} & =2 \ell(\ell+1)\left(\frac{1}{\xi} \partial_{\chi} H_{\xi}-\left[\partial_{\xi}-\frac{1}{\xi}\right] H_{n}\right), \\
P_{w} & =-\frac{\ell(\ell+1)(\ell(\ell+1)-2)}{2 \xi^{3} \cosh ^{2} \chi}\left(\partial_{\chi} w-2 \xi H_{n}\right) .
\end{aligned}
$$

The Hamiltonian is defined by

$$
\mathbf{H}^{(o)}=\sum_{\ell, m} \sum_{i} \overline{P_{i}^{(o) \ell m}}\left(\partial_{\chi} H_{i}^{(o) \ell m}\right)-\mathbf{L}^{(o)}
$$

with the replacement of $\partial_{\chi} H_{n}$ by $\lambda$. The canonical equations of motion are given by Eq. (A17).

We set the gauge condition

$$
H_{\xi}=0 .
$$

This gauge condition implies the consistency condition, $\partial_{\chi} H_{\xi}=0$, which becomes 


$$
0=D=\frac{P_{\xi}}{2 \ell(\ell+1)}+\left(\partial_{\xi}-\frac{1}{\xi}\right) H_{n}
$$

From the condition $\partial_{\chi} C=0$,

$$
P_{w}=\frac{\ell(\ell+1)}{\xi^{3}}\left[\frac{(\ell(\ell+1)-2)}{\cosh ^{2} \chi} \tanh \chi w+\hat{K}\left(\xi H_{n}\right)\right]
$$

follows. The second level consistency condition for the gauge condition, $\partial_{\chi} D=0$, reduces to

$$
\lambda=-2 \tanh \chi H_{n}-\frac{\ell(\ell+1)-2}{2 \xi \cosh ^{2} \chi} w .
$$

Now the second level consistency condition for the constraint equation, $\partial_{\chi}^{2} C=0$, is remaining. Again this condition is found to be satisfied by using the relations which we have already obtained. Thus we find that the system of the constraints closes. Now the equation for $Q^{(o) \ell m}:=\xi H_{n}^{(o) \ell m}$ reduces to

$$
\begin{aligned}
\partial_{\chi} Q & =\frac{\Pi}{\cosh ^{2} \chi}, \\
\partial_{\chi} \Pi & =\left[-\ell(\ell+1)-\cosh ^{2} \chi \hat{K}\right] Q,
\end{aligned}
$$

where

$$
\Pi^{(o) \ell m}:=-\frac{\ell(\ell+1)-2}{2} w^{(o) \ell m}-2 \xi \sinh \chi \cosh \chi H_{n}^{(o) \ell m} .
$$

All the other variables can be written in terms of $Q$ and $\Pi$ as

$$
\begin{aligned}
H_{n} & =\frac{Q}{\xi}, \\
H_{\xi} & =0, \\
w & =\frac{-2}{\ell(\ell+1)-2}[\Pi+2 \sinh \chi \cosh \chi Q], \\
P_{n} & =-\frac{4 \ell(\ell+1)}{\xi^{2}} \tanh \chi Q, \\
P_{\xi} & =-\frac{2 \ell(\ell+1)}{\xi}\left(\partial_{\xi}-\frac{2}{\xi}\right) Q, \\
P_{w} & =\frac{\ell(\ell+1)}{\xi^{3}}\left[\left(\hat{K}-4 \tanh ^{2} \chi\right) Q-\frac{2 \tanh ^{2} \chi}{\cosh ^{2} \chi} \Pi .\right.
\end{aligned}
$$

Of course, Eqs. A59 and A61 are consistent with the mode functions obtained in Section 3.

Substituting (A61) into the canonical form of the action the reduced action becomes

$$
\begin{aligned}
\int d \chi \int d \xi \mathcal{L}_{(r e d)}^{(o)}= & \sum_{\ell, m} \frac{2 \ell(\ell+1)}{\ell(\ell+1)-2} \int d \chi \int \frac{d \xi}{\xi^{3}} \\
& \times\left[\Pi \hat{K}\left(\partial_{\chi} Q\right)-\frac{1}{2}\left(\frac{1}{\cosh ^{2} \chi} \bar{\Pi} \hat{K} \Pi+\bar{Q} \hat{K}\left\{\ell(\ell+1)+\hat{K} \cosh ^{2} \chi\right\} Q\right)\right]
\end{aligned}
$$

As before, the variables $Q$ and $\Pi$ are expanded as

$$
Q^{(o) \ell m}=-\xi^{2} \int d p Q_{(o) p \ell m} \mathcal{U}_{p}, \quad \Pi^{(o) \ell m}=-\xi^{2} \int d p \Pi_{(o) p \ell m} \mathcal{U}_{p}
$$

Then the reality condition becomes

$$
\overline{Q_{p \ell m}}=Q_{-p \ell-m}
$$

Using this expansion, the reduced action becomes 


$$
\begin{aligned}
\int d \chi d \xi \mathcal{L}_{(r e d)}^{(o)}= & \int_{-\infty}^{\infty} d p \sum_{\ell, m} \frac{2\left(p^{2}+1\right) \ell(\ell+1)}{(\ell-1)(\ell+2)} \\
& \times \int d \chi\left[\overline{\Pi_{(o) p \ell m}}\left(\partial_{\chi} Q_{(o) p \ell m}\right)-\frac{1}{2}\left(\frac{\left|\Pi_{(o) p \ell m}\right|^{2}}{\cosh ^{2} \chi}+\left\{\ell(\ell+1)+\left(p^{2}+1\right) \cosh ^{2} \chi\right\}\left|Q_{(o) p \ell m}\right|^{2}\right)\right] .
\end{aligned}
$$

Comparison of the $\mathcal{H}_{\chi A}^{(e) p \ell m}$ component with the definition of $Q$ gives

$$
Q_{(o) p \ell m}=\mathcal{N}_{(o) p \ell m} \mathcal{T}_{5}^{p \ell}=\mathcal{N}_{(o) p \ell m} \mathcal{P}_{p \ell}
$$

Then, repeating the same procedure taken below Eq. (A37), we obtain the result given in Eq. 3.51).

[1] J. R. Gott III, Nature 295, 304 (1982).

[2] M. Bucher, A. S. Goldhaber and N. Turok, Nucl. Phys. B, Proc. Suppl. 43, 173 (1995); M. Bucher, and N. Turok, Phys. Rev. D 52, 5538 (1995).

[3] K. Yamamoto, M. Sasaki and T. Tanaka, Astrophys. J. 455, 412 (1995).

[4] A. D. Linde, Phys. Lett. B 351, 99 (1995).

[5] A. D. Linde and A. Mezhlumian, Phys. Rev. D 52, 5538 (1995).

[6] A. M. Green and A. R. Liddle, report No. SUSSEX-AST 96/7-7, astro-ph/9607166.

[7] S. Coleman, Phys. Rev. D 15, 2929 (1977).

[8] S. Coleman and F. De Luccia, Phys. Rev. D 213305 (1980).

[9] K. Yamamoto, T. Tanaka, and M. Sasaki, Phys. Rev. D 51, 2968 (1995).

[10] T. Hamazaki, M. Sasaki, T. Tanaka, and K. Yamamoto, Phys. Rev. D 532045 (1996).

[11] M. Sasaki, T. Tanaka, and K. Yamamoto, Phys. Rev. D 51, 2979 (1995).

[12] M. Sasaki and T. Tanaka, to appear in Phys. rev. D 54.

[13] K. Yamamoto, M. Sasaki and T. Tanaka, to appear in Phys. rev. D 54.

[14] K. Yamamoto and E. F. Bunn, Astrophys. J. 464, 8 (1996).

[15] J. Garriga, Report No. UAB-FT-387, gr-qc/9602025 (1996), (unpublished).

[16] J. Garcia-Bellido, Phys. Rev. D 54(1996).

[17] J. D. Cohn, Reprot No. LBNL-38560, UCB-PTH-96/10, astro-ph/9605132.

[18] D. Lyth and A. Woszczyna, Phys. Rev. D 523338 (1995).

[19] B. Allen and R. Caldwell, Report No. WISC-MILW-94-TH-21 (1994), (unpublished).

[20] A. diSessa, J. Math. Phys. 15, 1892 (1974).

[21] U. H. Gerlach, Phys.rev. D 28, 761 (1983).

[22] W. Magnus, F. Oberhettinger and R. S. Soni, Formulas and Theorems for the Special Functions of Mathematical Physics (Springer, New York, 1966), pp. 92.

[23] R. K. Sachs and A. M. Wolfe, Astrophys. J. 147, 73 (1967).

[24] K. Tomita, Prog. Theor. Phys. 68, 310 (1982). 